\title{
Excitonic Harvestation Radiative Recombination by Analysis and Comparison of the Experimental Data Measurements and the Theoretical Data Calculations from Silvaco-TCAD
}

Sakineh Akbari Nia ( $\square$ akbari2028@yahoo.com )

University of Mohaghegh Ardabili https://orcid.org/0000-0002-5677-289X

Shahriar Molamohammadi

University of Mohaghegh Ardabili

Yousef Seyed Jalili

Islamic Azad University Science and Research Branch

\section{Research Article}

Keywords: Organic semiconductor, OLED, exciton, Richardson-Schottky, field effect emission

Posted Date: February 18th, 2021

DOl: https://doi.org/10.21203/rs.3.rs-172368/v1

License: (c) (1) This work is licensed under a Creative Commons Attribution 4.0 International License.

Read Full License 


\title{
Excitonic Harvestation Radiative Recombination
}

\section{by Analysis and Comparison of the Experimental} Data Measurements and the Theoretical Data Calculations from Silvaco-TCAD

\author{
Sakineh Akbari Nia ${ }^{*}$, Shahriar Molamohammadi ${ }^{b}$, and Yousef Seyed Jalili ${ }^{c, d}$ \\ a University of Mohaghegh Ardabili, Ardabil, Iran, akbari2028@yahoo.com. \\ b University of Mohaghegh Ardabili, Ardabil, Iran
}

c Nano-Optoelectronics Laboratory, Sheykh Bahaee Research Complex,Science and Research Branch, Islamic Azad University, Tehran, Iran

d Nano-Optoelectronics Group, Physics Department, Science and Research Branch,Islamic Azad University, Tehran, Iran

\begin{abstract}
Organic Light Emitting Diodes (OLEDs) have developed to show many advantageous and noticeable properties including their flexibility, easy processing, very cost effective chemical technology, and easy combining of organic material, hence having optimized performance is very significant. Therefore, we have to find new methods in order to be able to use the total singlet and triplet excitons population and to achieve $100 \%$ internal efficiency. If we can extend the internal efficiency to $100 \%$, this will lead to efficient production of OLEDs in the industry. In this work, to consider increasing excitons' population, we have worked on the uniformity of the Electron Transport Layer (ETL) and Emissive Layer (EL) by applying argon atmosphere pressure cold jet plasma. Examining the experimental data in comparison with the theoretical data has shown us that having a better uniformity in barriers (based on AFM images) and decreasing contact angle will lead to a better injection and more exciton's population leading to an increase in the internal efficiency.
\end{abstract}

KEY WORDS: Organic semiconductor, OLED, exciton, Richardson-Schottky, field effect emission.

\section{INTRODUCTION}

In recent decades, Organic Light Emitting Diodes (OLEDs) have been considered by many researchers because of their flexibility, ease of processibility, very cost effective chemical technology, and easy combining of organic material [1-2]. If these diodes are well optimized and have a good internal efficiency $\left(\eta_{i n}\right)$ and in turn a good external efficiency, then OLEDs will be the dominant light source because of their cost effectiveness. In order to optimize OLEDs, a lot of researches have been done. In OLEDs, after injecting electron and hole from opposite electrodes into the emissive layer, two kinds of excitons would be formed: the singlet exciton and the triplet 
exciton [2-4]. Singlet excitons which are spin-allowed can only contribute to about $25 \%$ of the internal efficiency, and triplet excitons which are spin-forbidden can contribute to about $75 \%$ of it [4-6], depending on the population of each one respectively, therefore increasing the exciton's population attracts considerable attention. If the triplet excitons cannot recombine due to forbidden spin configuration, then the light emission can occur through increased singlet excitons, and this can increase the light-out efficiency $\left(\eta_{\text {out }}\right)[5]$.

According to different studies, there are some ways to increase quantum efficiency based on excitons. For example, as Cao et al have shown, one way is with increasing the cross section of an electron-hole pair to form excitons [7-8]. The other way is to harvest the radiation emission from all of the excitons, singlets and triplets[10]. Here, in this paper, our aim is achieving better efficiency by increasing the population of the excitons. In order to consider whether excitons' population are increased or not, first, we can work on barriers and optimize them to have better injection and hence more excitons would be created [1,9-10], and second, we can dope some materials into our organic layers or add other organic layer as a hole transport layer, electron transport layer, hole or electron blocker, and so on [11-13]. But, as adding various layers make it difficult to reproduce the device, it is best to enhance a device with fewer layers. In this paper we will study optimizing the barrier surfaces. Thus, in this work, we will attempt to consider the change in the number of excitons and its effect on MEH-PPV based OLEDs behaviors in the presence of the effect of the argon atmospheric pressure cold jet plasma on the ITO layer.

\section{EXPERIMENTAL SETUP}

In order to make the OLEDs, many experimental processes had been utilized. In fact, these multilayer devices include ITO(150nm)/PEDOT:PSS(30nm)/MEH-PPV(60nm)/Ag(100nm). First, Petri dishes were cleaned by dishwasher liquid, acetone, ethanol, and rinsed thoroughly by deionize water, respectively. After that, ITO(Indium Tin Oxide) layers, as the transparent conductive layers, were placed within the cleaned Petri dishes and were washed by dishwasher liquid for one minute, and then by acetone, ethanol, and deionize water, respectively for 10 minutes in ultrasonic. In order to dry, anneal, and remove some probable remaining contaminants, the ITO layers were put in the oven, under 180 degree centigrade, for $30 \mathrm{Min}$. After this time, the ITO layers were ready for deposition of other layers. Before coating the next layer, the primed substrates were masked and according to table 1, argon atmosphere pressure cold jet plasma was applied on them. It is noticeable that the flame's distance from substrates was $0.5 \mathrm{~cm}$. Then, in order to build the multi-layer polymer diodes, and to investigate the effect of the argon atmosphere pressure cold jet plasma on the excitons, and the diodes behavior, poly $(3,4-$ ethylenedioxythiophene):poly(styrene sulfonate) (PEDOT:PSS)( 6\% PEDOT:PSS solution in ethylene glycol) as a hole transport layer and poly [1 - methoxy - 4 - ( 20 - ethylhexyloxy ) - p phenylenevinylene ] (MEH-PPV)( 5g/L MEH-PPV solution in chloroform) as the emissive layer were spin coated on the ITO layer respectively. These solutions were deposited by spin coating inside the glove box respectively for each device and were dried under the conditions given in Table 2. After depositing and drying PEDOT:PSS layer, all of devices were put under the argon atmosphere pressure cold jet plasma for one minute. Finally, an Ag layer as the cathode was 
evaporated on the previous polymeric layer, Table 2. During the device fabrication, required measurements analysis such as the sheet resistance for ITO and PEDOT:PSS layers, Figure 1 and 2, the contact angle, Figure 3, Fourier Transform Infrared Spectroscopy (FTIR), Figure 4 and 5 , and Atomic Force Microscopic (AFM), Figure 6 and 7, were prepared. Also, at the end of the fabrication process, I-V measurements analysis were carried out to study injection, excitons behavior and recombination.

Table 1. Plasma setting (for argon atmosphere pressure cold jet plasma device with ref $=0$ and watt $=12$ ) for ITO substrates and PEDOT:PSS.

\begin{tabular}{|l|l|l|l|l|}
\hline Device number & $\mathrm{D}_{1}$ & $\mathrm{D}_{2}$ & $\mathrm{D}_{3}$ & $\mathrm{D}_{4}$ \\
\hline ITO & $0 \mathrm{~min}$ & $9 \mathrm{~min}$ & $11 \mathrm{~min}$ & $13 \mathrm{~min}$ \\
\hline PEDOT:PSS & $1 \mathrm{~min}$ & $1 \mathrm{~min}$ & $1 \mathrm{~min}$ & $1 \mathrm{~min}$ \\
\hline
\end{tabular}

Table 2. Deposition and dry settings for PEDOT:PSS, MEH-PPV, and Ag.

\begin{tabular}{|l|l|l|l|}
\hline Spin coat deposition settings & Volume $(\mu \mathrm{l})$ & Time $(\mathrm{s})$ & Rpm \\
\hline Material & 10 & 60 & 3000 \\
\hline PEDOT:PSS & 20 & 30 & 3500 \\
\hline MEH-PPV & \multicolumn{3}{l|}{} \\
\hline Evaporation setting & Volume(gr) & Time(s) & Vacuum $(\mathrm{Pa})$ \\
\hline Material & 10 & $5^{*} 10^{-1}$ \\
\hline Ag & 5 & \multicolumn{2}{l|}{} \\
\hline Drying settings & Sdl odq' st qd' $\left.{ }^{\circ} \mathrm{C}\right)$ & Time(min) \\
\hline Material & $180($ oven $)$ & 7 \\
\hline PEDOT:PSS & $100($ heater $)$ & 90 \\
\hline MEH-PPV &
\end{tabular}

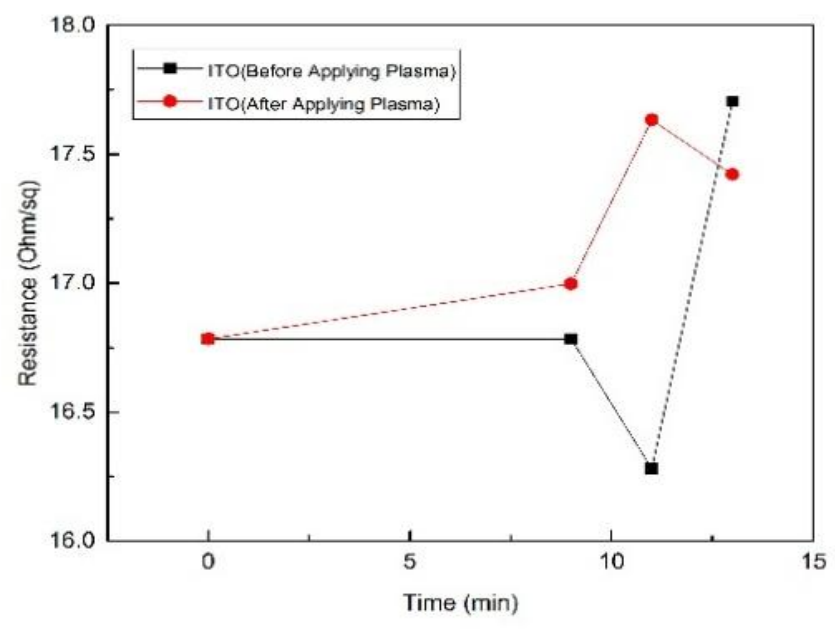


Figure 1. Sheet resistance curve for ITO layers before applying argon atmosphere pressure cold jet plasma, full red circle, and after applying argon atmosphere pressure cold jet plasma, full black circle.

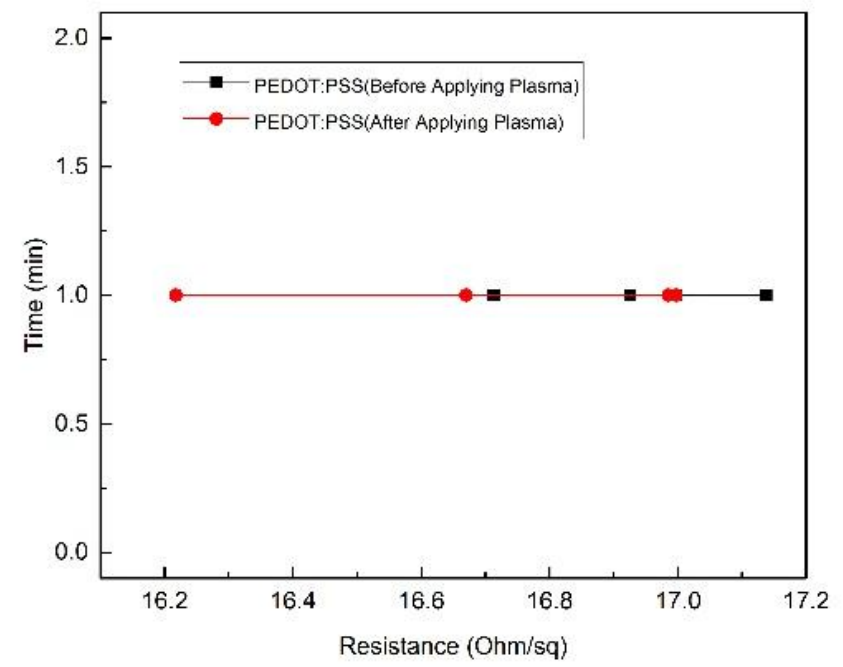

Figure 2. Sheet resistance curve for PEDOT:PSS layers before applying argon atmosphere pressure cold jet plasma, full red circle, and after applying argon atmosphere pressure cold jet plasma, full black circle.

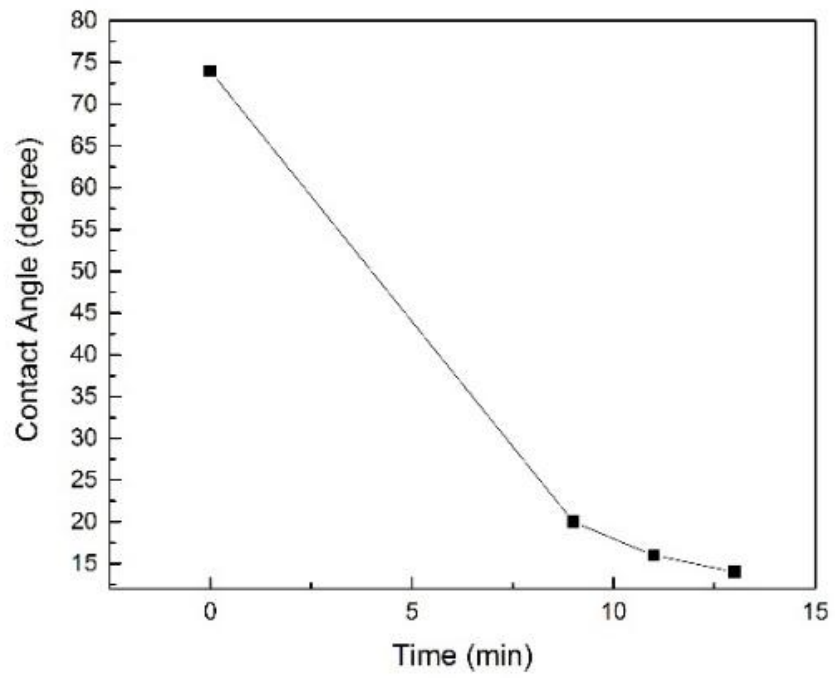

Figure 3. CCD images: Contact angle reducing under applying argon atmosphere pressure cold jet plasma for ITO substrates. 


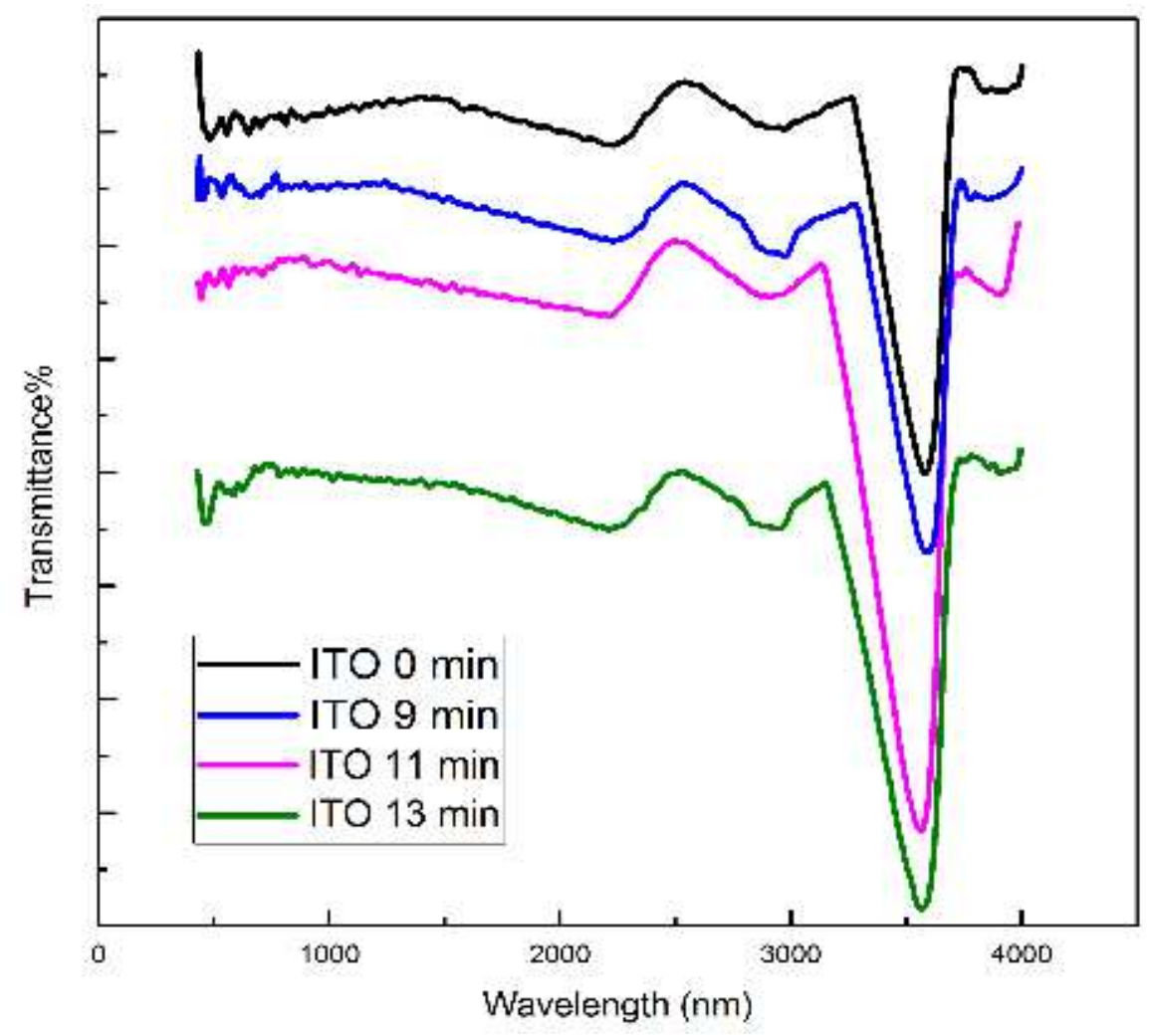

Figure 4. FTIR measurement analyses for ITO layers in various plasma application time.

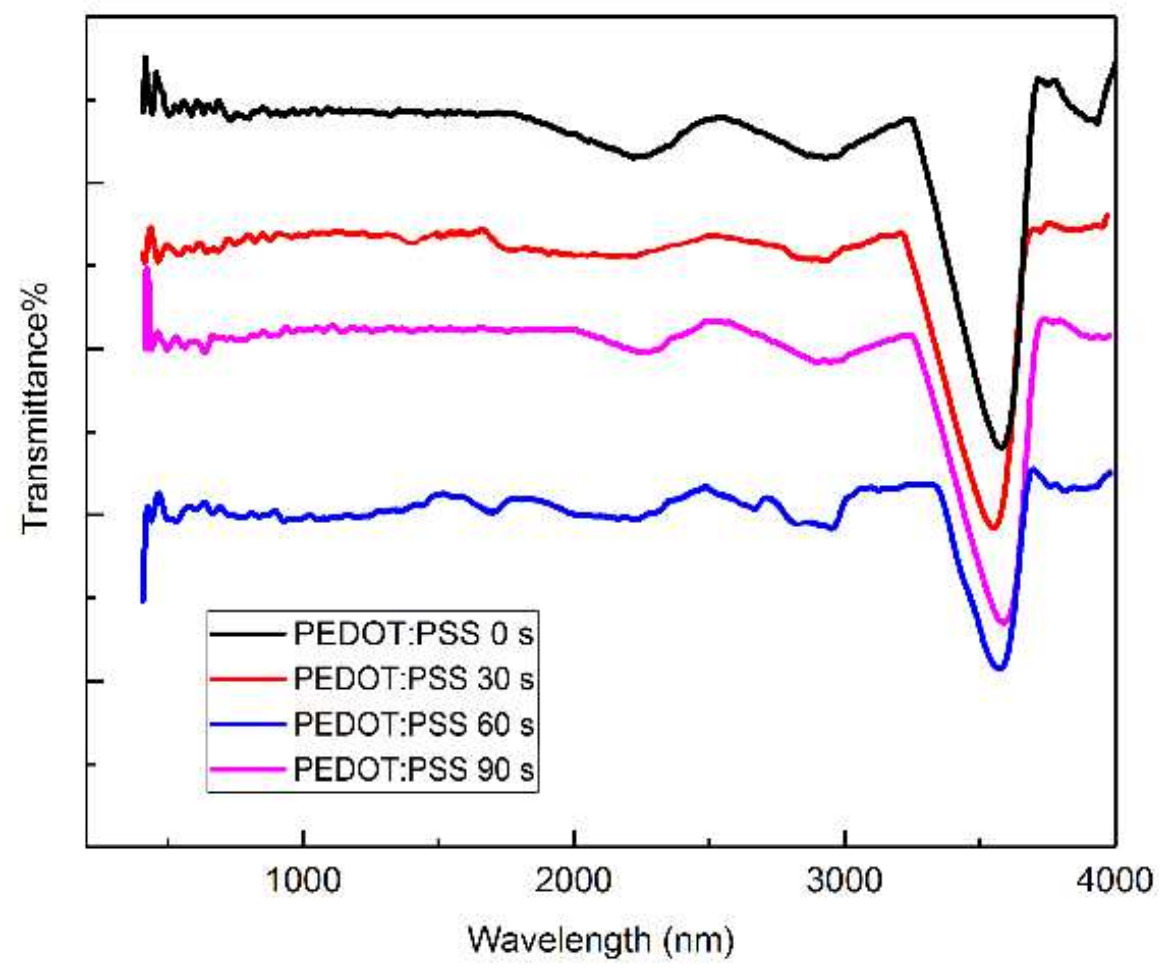

Figure 5. FTIR measurement analyses for PEDOT:PSS layers in various plasma application time. 

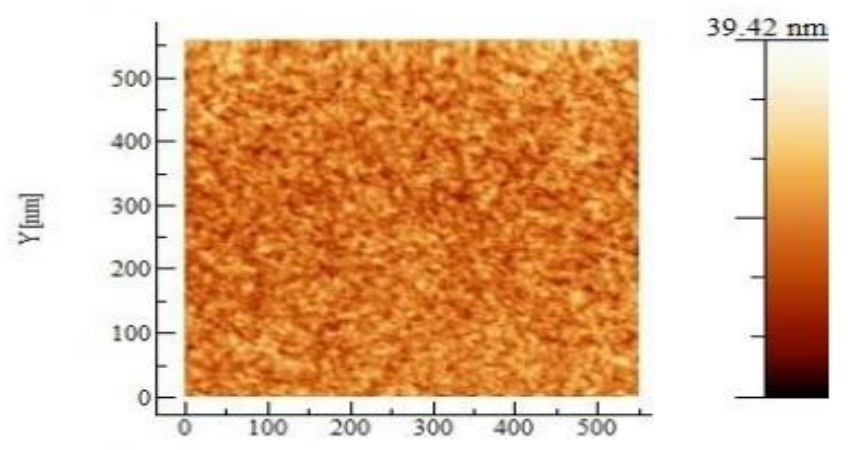

A) ITO(0 min plasma)
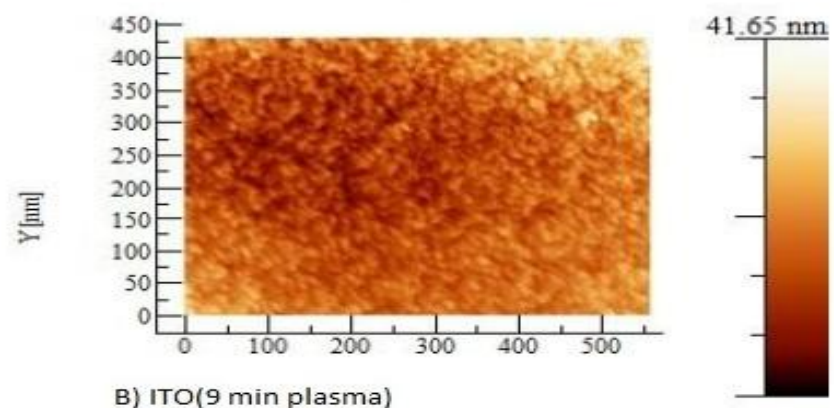

B) ITO(9 min plasma)
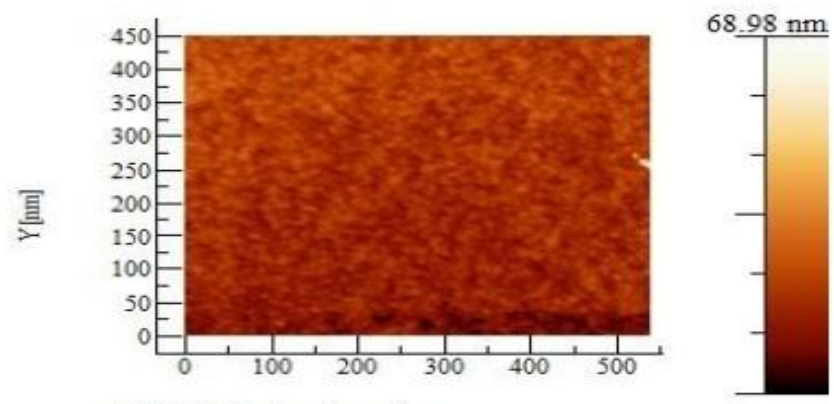

C) ITO(11 min plasma)
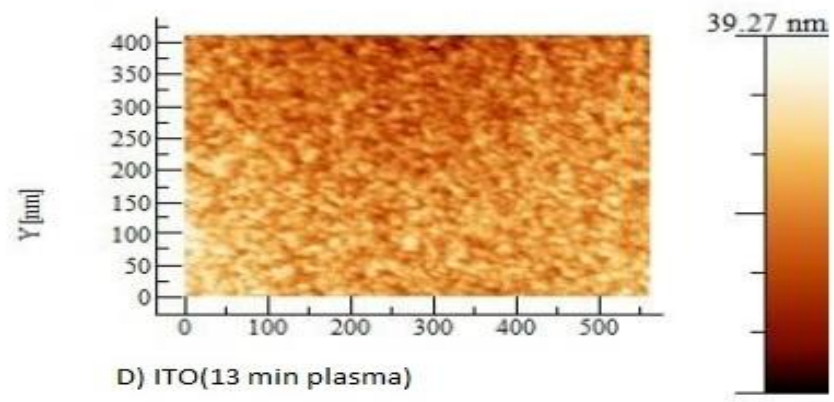

D) ITO(13 min plasma)

$\mathrm{X}[\mathrm{nm}]$

Figure 6. AFM analyses for ITO surface after applying A) 0 minute, B) 9 minute, C) 11 minute, and D) 13 minute argon atmosphere pressure cold jet plasma. 
(A)
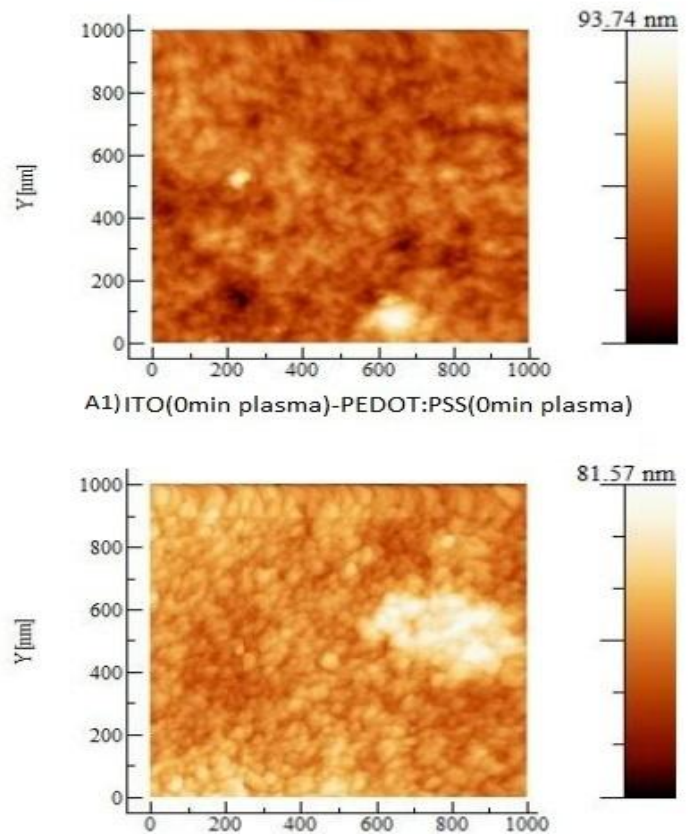

A2) ITO(9min plasma)-PEDOT:PSS(Omin plasma)

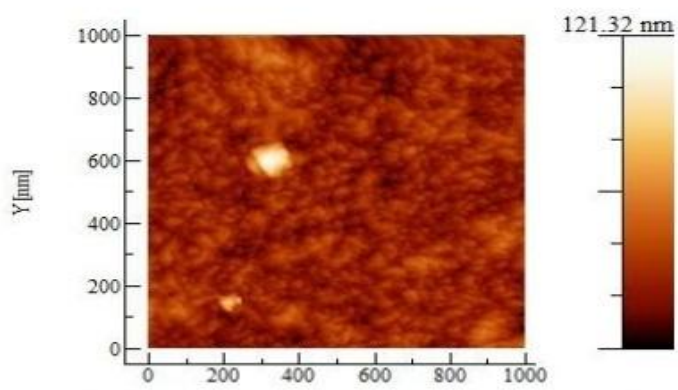

А3) ITO(11 min plasma)-PEDOT:PSS(Omin plasma)

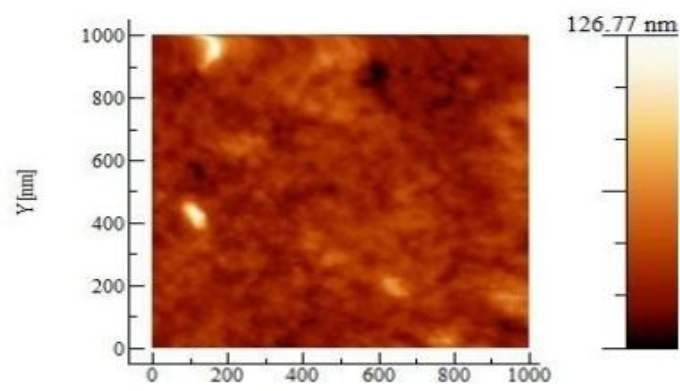

A4) ITO(13min plasma)-PEDOT:PSS(Omin plasma)

$\mathrm{X}[\mathrm{nm}]$
(B)

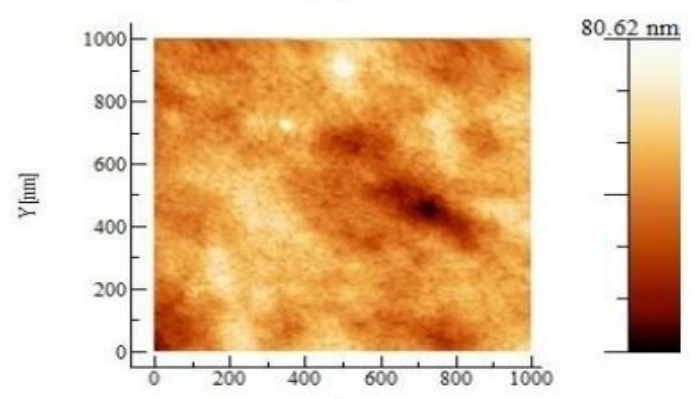

B1) ITO(Omin plasma)-PEDOT:PSS(1 min plasma)

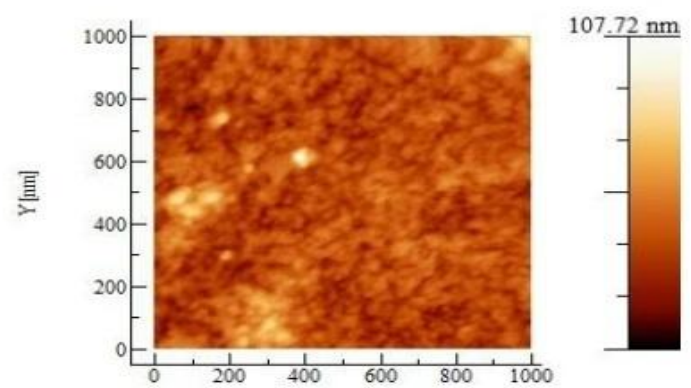

B2) ITO(9min plasma)-PEDOT:PSS(1 min plasma)

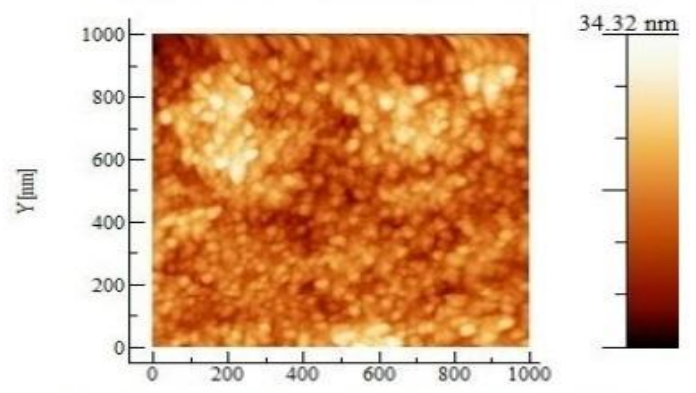

В3) ITO(11 min plasma)-PEDOT:PSS(1 min plasma)

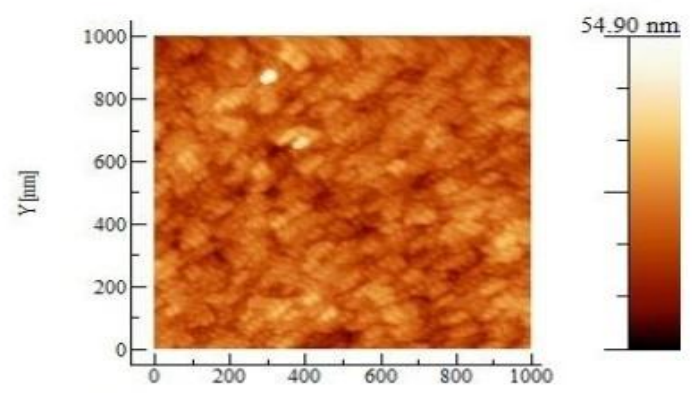

B4) ITO(13min plasma)-PEDOT:PSS(1 min plasma)

$\mathrm{X}[\mathrm{nm}]$

Figure 7. AFM analyses for PEDOT:PSS surface before, A1-A4, and after, B1-B4, applying 1 minute argon atmosphere pressure cold jet plasma. 


\section{MODELING}

Simulation modeling of optoelectronic device structures prior to device fabrication helps to save and conserve materials and device fabrication costs. In this section we will compare our simulation results with the performance measurements of our fabricated devices. However, it is important to acquire and know the essential input data. In here, they were collected from among the related authentic articles [14-17]. Therefore, the OLED structure was simulated in 2-D ATLAS simulator of Silvaco Co. that provides general capabilities for accurate simulation of organic device structures. At first, we defined a mesh of structure, the determination of a good mesh is very important for accuracy and numerical efficiency of the simulation model. Thus, we allocated a fine grid only in critical areas where the recombination occurs and a coarser grid elsewhere in order to get an accurate and efficient simulation. Once the mesh was defined, we assigned a material type of every part of the device using ITO, PEDOT:PSS, MEH-PPV, and Ag. Finally, we specified the electrodes location and the doping level within each region. The simulation results are illustrated in figures 8-10 showing the device structure, Langvine recombination rate on different place of the device, and the Longvine recombination curve respectively.

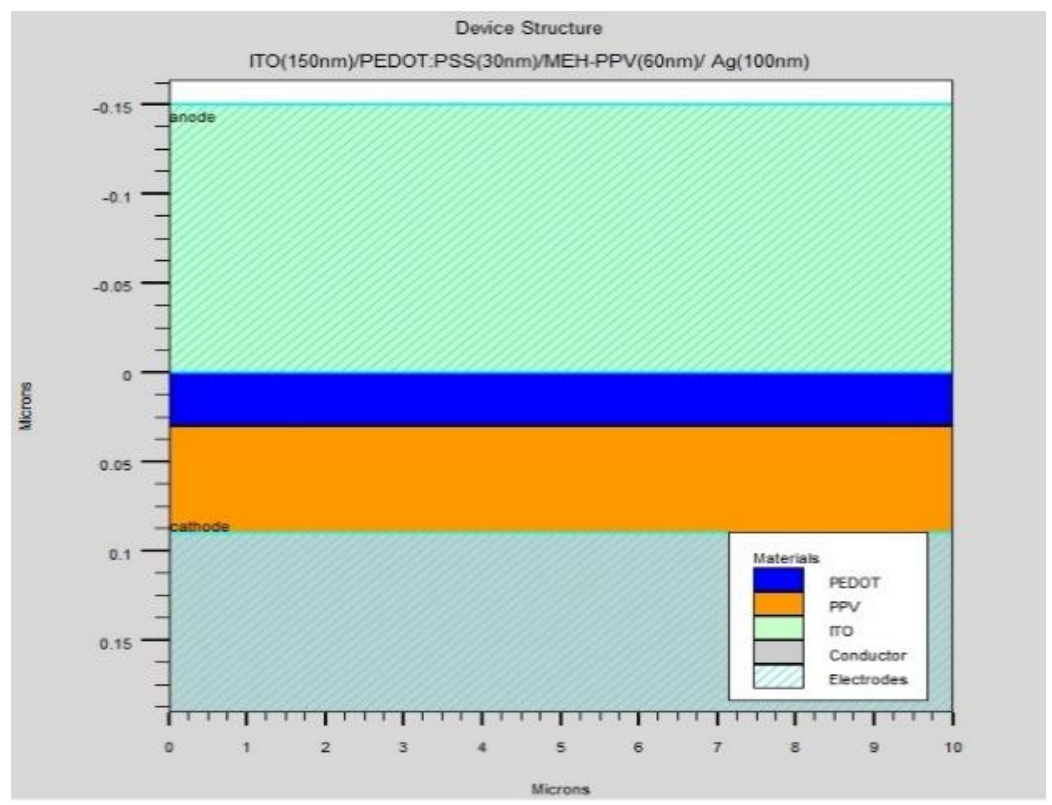

Figure 8. The Device structure consisting of the ITO(150nm)/PEDOT:PSS(30nm)/ MEH-

$$
\operatorname{PPV}(60 \mathrm{~nm}) / \mathrm{Ag}(100 \mathrm{~nm}) \text {. }
$$




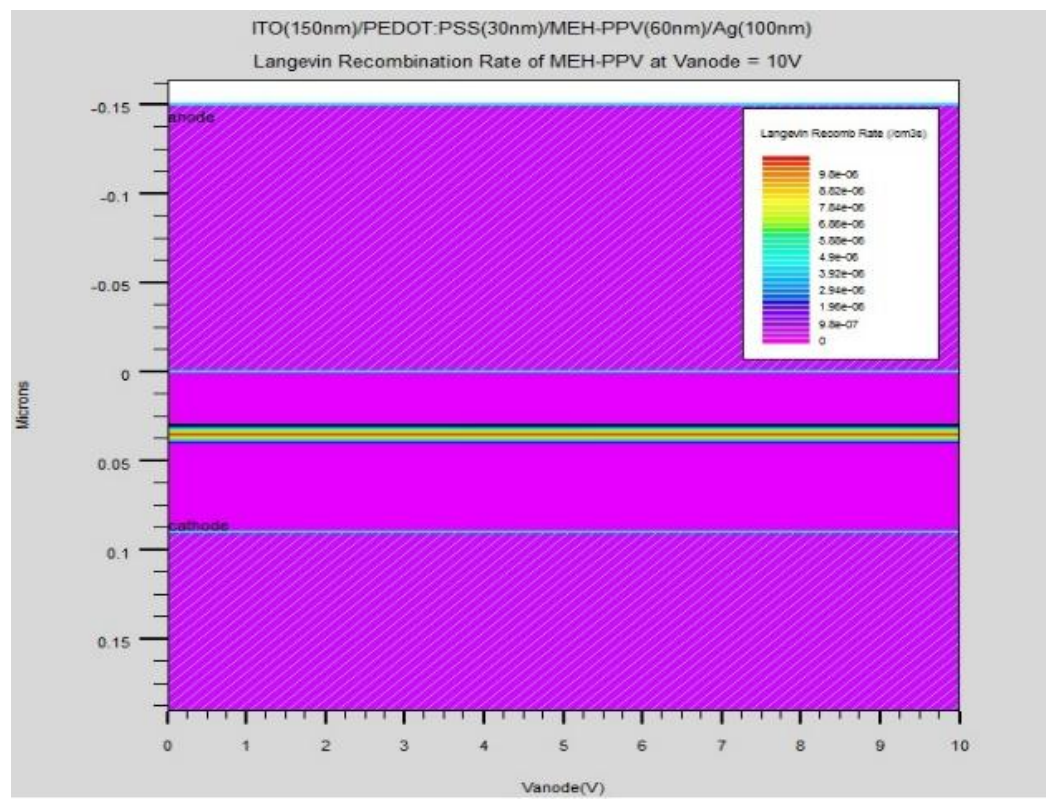

Figure 9. Langvin recombination rate along the various points of the device.

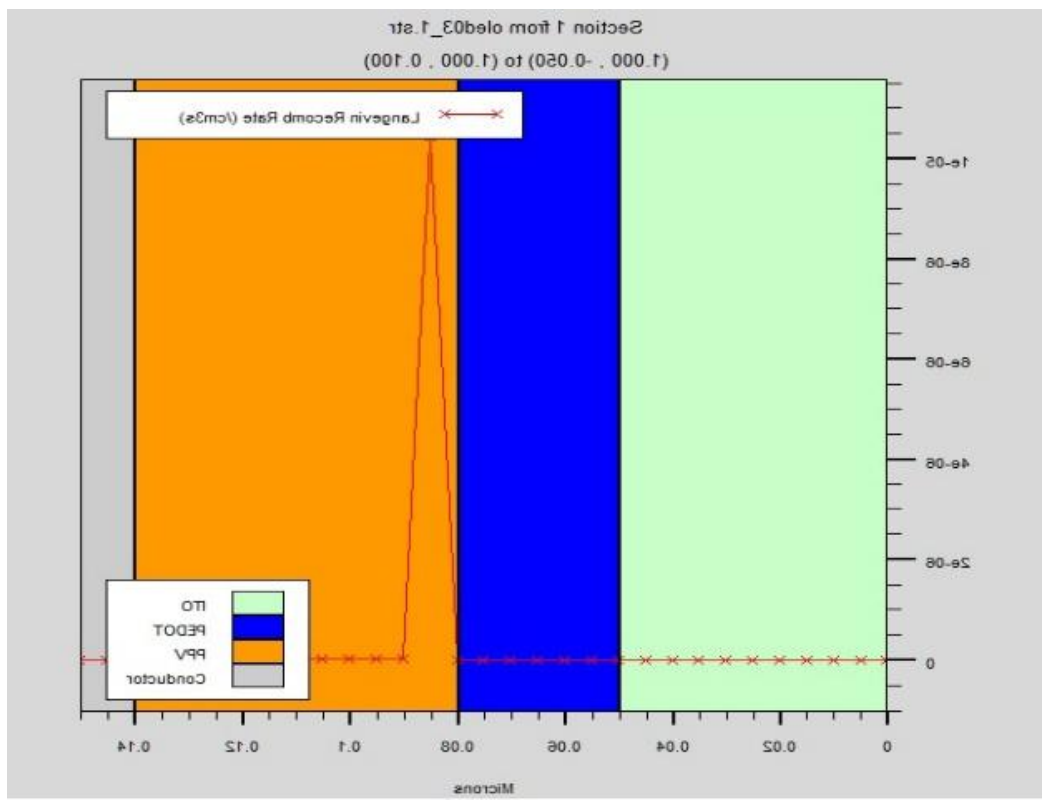

Figure 10. Longvin recombination rate curve in deferent layers of the device.

Longvine recombination curve, Figure 10 shows that the carrier recombination occurs near the barrier surface. Besides, the I-V data was also extracted from Silvaco TCAD simulation and was plotted by Origin software. The simulated I-V curve and the measured I-V curve of the fabricated devices have been illustrated in Figure 11. 


\section{RESULTS AND DISCUSSION}

For increasing the internal quantum efficiency, various studies have been carried out. The performance of an OLED can be optimized by finding the optimum emitting material(s), manipulating the charge carrier balances and location of the recombination zone and energy transfer [18]. For instance, in our work, applying Ar atmosphere cold plasma jet on ITO layer and PEDOT:PSS had lead to generation of more excitons within the emissive layer.

To investigate the ITO and PEDOT:PSS surface properties change, we measured the sheet resistance and carried out AFM measurements as well as FTIR analyzes. Also, the effects of these changes on current-voltage characteristic in the OLEDs were investigate for four two layer OLEDs and were compared with the corresponding device simulation models output data. Sheet resistance curves measured by four point probe are shown in Figure 1 for ITO layers, and Figure 2 for PEDOT:PSS layers. These diagrams indicate that the changes in the surface resistance were negligible under the applied plasma. As for the ITO layers, with the exception of the 13-minute plasma, we have observed a slight increase in resistance. But in the case of the PEDOT:PSS layers, generally, we see that the resistance is slightly reduced.

FTIR and AFM analysis, before and after application of the plasma, have shown chemical and physical changes. Our goal of using the cold plasma was surface roughness uniformity, so based on FTIR diagram, the plasma application time is chosen so that the surface chemistry does not change too much. Also according to Figure 4, presence of functional groups such as $-\mathrm{OH}, \mathrm{C}=\mathrm{C}$ and C-C bonds on ITO surface is conclusive, respectively at 3500, 1300, $1500 \mathrm{~nm}$. Change in height, width and minor changes in peak position represent the bond angle and bond length. Peak's stability suggests that because of low-power and low-pressure of the cold plasma applied, the plasma is not able to create new functional groups on the ITO surface. In the case of the PEDOT:PSS, FTIR diagram, Figure 5, has shown that, totally, changes in peak places, the wide of the peaks, and changes in their high is not noticeable. Also it has shown that the peaks had not been eliminated nor created. These results state that the application of low power and time plasma has maintained the surface chemistry well. So in this layer, surface compounds are very much preserved. In fact, for this layer, the bond angles and bond lengths have changed which has ultimately led to border uniformity.

CCD ITO images, taken after applying the cold plasma, Figure 3, show that the contact angle has strikingly decreased. Since these layers are hydrophobic, reducing the contact angle reduces their hydrophobicity. As a result, it is easier to deposit the next layer. Doing so will create a better contact surface between the two layers.

AFM analysis for ITO, Figure 6, show that the effect of plasma till 11 minute has led to surface uniformity, but after that, the 13 minute, the AFM image is similar to the picture for no-plasma ITO surface. Figure 7 shows the AFM images for PEDOT:PSS, before and after the plasma treatment. These AFM images indicate that the surface roughness and hydrophilicity after the plasma treatment was increased. These images indicate that ITO surface uniformity causes PEDOT:PSS surface uniformity, Figure 7. A Also, according to images in Figure 7.B, clearly after one minute of plasma application, the uniformity of the layer was further enhanced. 
Our goal of working on the barriers was to increase the contact level of the layers and, consequently, to increase the carrier injection and hence increase the excitons. Therefore, to consider injection, the Voltage-current characteristic, as a powerful tool to study relaxation processes, charge transfer mechanisms and metal/organic behaviors, will be illustrated. In our last essay, we had made some single and multi-layer devices, and in our investigation we understood that by applying the plasma on multi-layer devices both the Ohmic junction and the Space Charge Limited Currents (SCLC) current were improved [10]. Here the I-V curve were considered based on Richardson-Schottky Thermionic Emission, and Fowler-Nordheim Tunneling Effect models for all of the four multi-layer OLEDs plus a simulated device.

We would point out that based on Figure 11, increasing the plasma application time, the starting voltages had decreased. Also, the curve D3 matches quite well with the simulated device's I-V curve, meaning that D3 is closer to the ideal case of the simulated device.

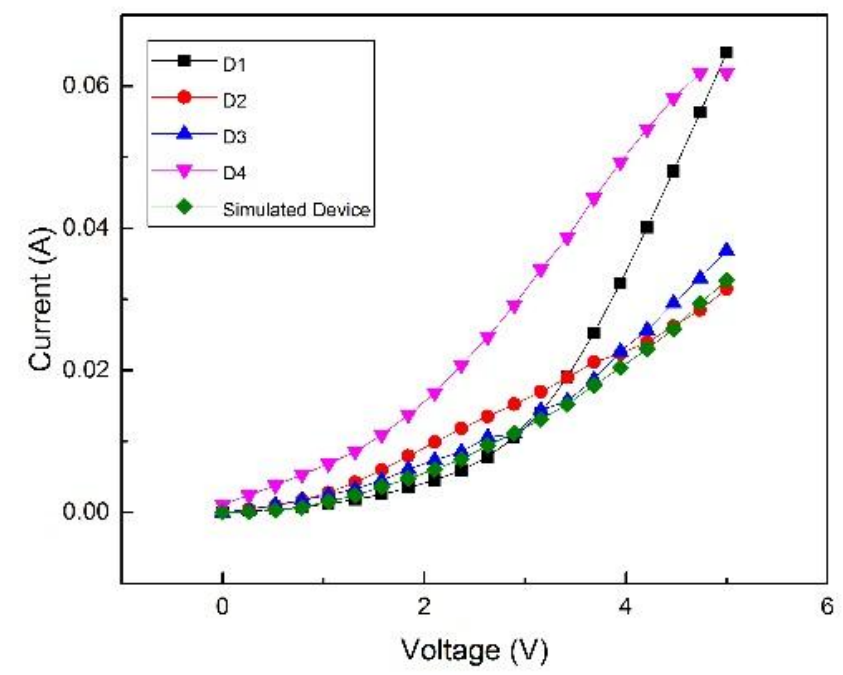

Figure 11. Current-Voltage characteristics of devices D1, D2, D3, D4, and simulated device with the variation plasma application time. 


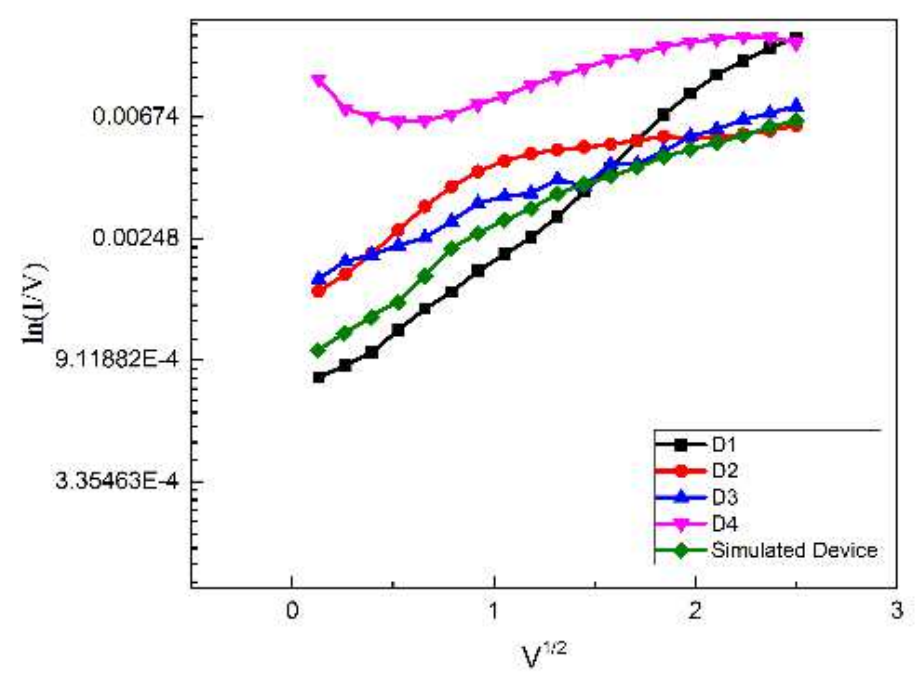

Figure 12. Dependence of the current density on the applied voltage in D1, D2, D3, D4, and the simulated device, plotted according to the Richardson-Schottky Thermionic Emission model.

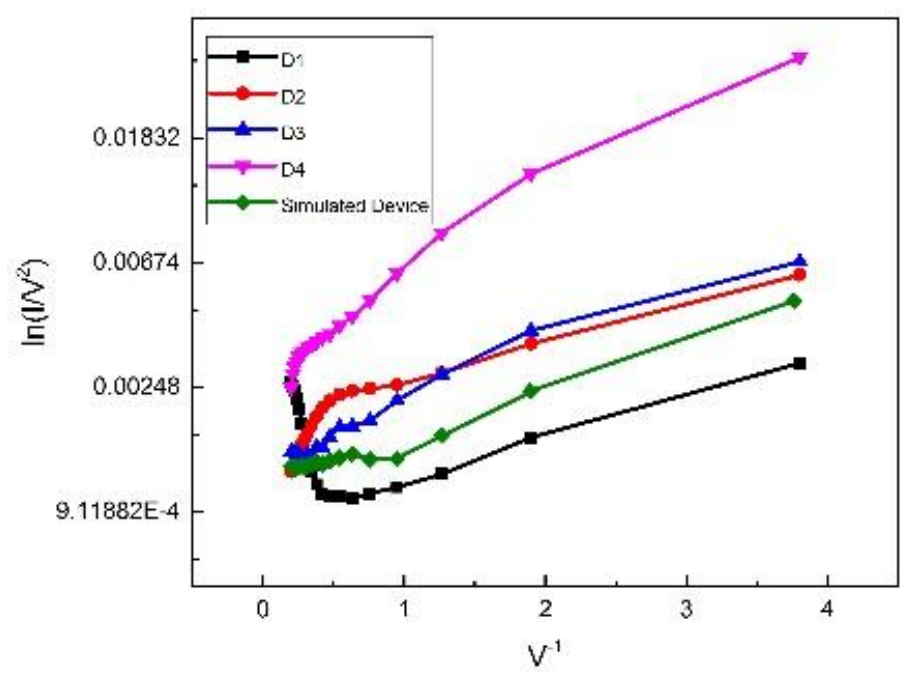

Figure 13. Dependence of the current density on the applied voltage in D1, D2, D3, D4, and simulated device, plotted in the Fowler-Nordheim tunneling emission model.

Now, for investigating the injection properties, the current-voltage characteristics curve could be divided into two regions. The region with low voltages follows the Richardson-Schottky Thermionic Emission model. The main suppose in this model is that charge carriers have enough heat to overcome the potential barrier [19-20]. 
In this model, current density is shown by "Equation 1"

$$
J=q N_{V} \mu(T) F \exp \left(\frac{-\varphi_{b}-\Delta \varphi}{\mathrm{KT}}\right)
$$

Where $\mathrm{q}$ is the basic charge, $\mathrm{N}_{\mathrm{V}}$ the effective density of states, $\mu(\mathrm{T})$ the temperature dependent mobility, $\mathrm{F}$ the electrical field, $\varphi_{\mathrm{b}}$ the energy barrier height at zero applied electrical field, $\Delta \varphi$ the change in the height of the energy barrier under the influence of image charge force and the applied electric field, $\mathrm{K}$ the Boltzmann constant, and $\mathrm{T}$ is the temperature. In this model $\ln (\mathrm{J} / \mathrm{V})$ via $\mathrm{V}^{1 / 2}$ gives a straight line [21-22]. Figure 12 illustrates the Richardson-Schottky Thermionic Emission curve. Considering the right half of the diagram (the low voltage region), we can see that the Schottky injection increases with increasing the plasma application time, but for D4, the diagram has an inappropriately shape. The curve with no-plasma has the least Schottky injection, and Schottky injection for D3 is the most similar to the simulated device. As all the other parameters of structures are constant, according to "Equation 1", injection increase must be because of workfunction reduction indicating that we can treat the surface as an electrically ohmic contact. Therefore, also considering that PEDOT:PSS acts as the electron blocking layer (EBL) too, charge carriers would be confined in the emissive layer (EL). This increases the recombination and the number of excitons in this layer.

The other region relates to applied voltages over 3.5 V. This region will be considered by FowlerNordheim tunneling effects [23]. According to this model, when the applied voltage increases, the depth of the triangular barrier decreases, then it will be appropriate for tunneling. In this model, current density for triangular barrier is shown by "Equation 2" [24].

$$
\mathrm{J}_{\mathrm{FN}}=\mathrm{AF}^{2} \mathrm{e}^{-\mathrm{F}_{0} / \mathrm{F}}
$$

Where $\mathrm{A}$ and $\mathrm{F}_{0}$ are related to the potential barrier and are defined by "Equation 3 and 4".

$$
\begin{aligned}
& A=\frac{q^{3}}{8 \pi h \varphi_{B}} \\
& F_{0}=\frac{8 \pi \sqrt{2 m^{*} \varphi_{B}^{3}}}{3 q h}
\end{aligned}
$$

Where $\mathrm{h}$ is the potential barrier height and $\mathrm{m}^{*}$ the carrier effective mass. In this model, $\operatorname{Ln}\left(\mathrm{J} / \mathrm{V}^{2}\right)$ via $\mathrm{V}^{-1}$ gives a straight line [24]. The left half part of the curves in Figure 13 relate to devices in high voltage region, for both the fabricated devices and the simulated device. So that, according to Figure 13, when the argon plasma application time increases, we get a better line fit. However for the device with no-plasma we do not have such a good line fit. This indicates that with increasing in the argon plasma application time, the thickness of the triangular barrier decreases further than before.

Finally, as the OLED's efficiency depends on injection, transport, and recombination, with increasing in the injection in both low and high voltage regions, the OLED's efficiency will also increase. 


\section{CONCLUSIONS}

In general, because of the hydrophobic film property of ITO and the hydrophilic film character of PEDOT:PSS, the contact angle between PEDOT:PSS and ITO is very large. This issue can interrupt the injection process and decrease the exciton's number, so that plasma with different application times was applied on ITO surfaces and decreased the contact angle. Further, surface uniformity is another important issue to have an appropriate injection and the application of the Ar atmospheric jet plasma was an apt choose to solve this problem. According to AFM images, by applying the plasma apt uniformity in the surfaces for 11 minute plasma application time for ITO and 1 minute argon plasma application time for PEDOT:PSS was achieved. By applying this modified layer in OLEDs, the injected carriers increased, hence leading to an increase in the excitons formation and the recombination rate. Increasing the charge injection rate results in the accumulation of charges near the electrode, and creates image charges. Increasing the image charges reduces the metal/polymer potential barrier, resulting in an increase in the injection.

\section{CKNOWLEDGEMENTS}

The authors would like to thank professor J. sing for his valuable guidance and discussion.

\section{REFERENCFES}

[1] R. Mahdiyar and M.R. Fadavieslam, "The effects of chemical treatment on ITO properties and performance of OLED devices", Opt Quant Electron, 52, Article number: 262(2020).

[2] S. Zou, Y. Shen, F. Xie, J. Chen, Y. Li and J. Tang, "Recent advances in organic light-emitting diodes: toward smart lighting and displays" Mater. Chem. Front., 4, 788-820(2020).

[3] J. Singh, "Study of organic light emitting devices (OLEDs) with optimal emission efficiency", Phys. Status Solidi C, 7, 984-987(2010).

[4] J. Singh, "Capturing triplet emission in white organic light emitting devices", Phys. Status Solidi A, 208, 1809-1812(2011).

[5] C. Adachi, M. A. Baldo, M.E. Thompson, and S. R Forrest, " Nearly 100\% internal phosphorescence efficiency in an organic light emitting device" Journal of Applied Physics, 90, 5048-5051 (2001).

[6]U. Shakeel, J. Singh, "Study of processes of reverse intersystem crossing(RISC) and thermally activated delayed fluorescence (TADF) in organic light emitting diodes (OLEDs)", ELSEVER, Organic Electronics, 59, 121-124(2018).

[7] Y. Cao, I.D. Parker, G. Yu, C. Zhang, A.J. Heeger, "Improved quantum efficiency for electroluminescence in semiconducting polymers" Nature, 397, 414-417(1999).

[8] Z. Shuai, D. Beljonne, R.J. Silbey, J.L. Bredas, "Singlet and Triplet Exciton Formation Rates in Conjugated Polymer Light-Emitting Diodes" Phys. Rev. Lett., 84, 131(2000). 
[9] H. Zheng, F. Zhang, N. Zhou, M. Sun, X. Li, Y. Xiao, Sh. Wang, "Self-assembled monolayermodified ITO for efficient organic light-emitting diodes: The impact of different self-assemble monolayers on interfacial and electroluminescent properties", ELSEVIER, Organic Electronics, 56, 89-95(2018).

[10] S. Akbari Nia, Y. Seyed Jalili, and A. Salar Elahi, "Application of argon atmospheric cold plasma for indium tin oxide (ITO) based diodes", AIP Advances 7, 095210 (2017).

[11] W. H. Lee, D. H. Kim, P. J. Jesuraj, H. Hafeez, J. Ch. Lee, D. K. Choi, T. S. Bae, S. M. Yu, M. Song, Ch. S. Kim and S. Y. Ryu, "Improvement of charge balance, recombination zone confinement, and low efficiency roll-off in green phosphorescent OLEDs by altering electron transport layer thickness", IOP, Materials Research Express, 5, Number 7(2018).

[12] L. Sh. Yang, H. F. Meng, Y. F. Chang, Ch. H. Lien, H.W. Zan, Sh. F. Horng, L. Duan, Y. Qiu, and C.W.Luo, "Stable and efficient blue fluorescent organic light-emitting diode by blade coating with or without electron-transport layer", ELSEVIER, Organic Electronics, 51, 615(2017).

[13] R. Kandulna, and R. B. Choudhary, "Robust electron transport properties of PANI/PPY/ZnO polymeric nanocomposites for OLED applications", ELSEVIER, Optik, 144, 40-48(2017).

[14] P.G. Da Costa, E. Conwell, "Excitons and the band gap in poly (phenylene vinylene)", Phys. Rev. B, 48, 1993-1996(1993).

[15] Y.F. Li, Y. Cao, J. Gao, D.L. Wang, G. Yu, A.J. Heeger, "Electrochemical properties of luminescent polymers and polymer light-emitting electrochemical cells", Synth. Met., 99, 243-248 (1999).

[16] K. Bhargava, A. Bilgaiyan, and V. Singh, "Two Dimensional Optoelectronic Simulation Based Comparison of Top and Bottom Contact Organic Phototransistors", Nanoscience and Nanotechnology, 15, 1-9(2015).

[17] S. M. H. Rizvi, P. Mantri, and B. Mazhari, "Traps signature in steady state current-voltage characteristics of organic diode", AIP, Journal of Applied Physics, 115, 244502 (2014).

[18] J. Kido, K. Hongawa, K. Okuyama, K. Nagai, "White light-emitting organic electroluminescent devices using the poly( $\mathrm{N}$-vinylcarbazole) emitter layer doped with three fluorescent dyes", AIP, Appl. Phys. Lett., 64, 815-817(1994).

[19] Sh. Hameed, P. Predeep and M.R. Baiju, "polymer light emitting diodes - a review on materials and techniques", Rev. Adv. Mater. Sci., 26, 30-42(2010).

[20] V. I. Arkhipov, E. V. Emelianova, Y. H. Tak, and H. Bässler, "Charge injection into lightemitting diodes: Theory and experiment" Appl. Phys., 84, 848-856(1998).

[21] P. R. Emtage, J. J. Odwyer, "Richardson-Schottky Effect in Insulators", Phys. Rev. Lett., 16, 356-358(1966). 
[22] P.W.M. Blom and M.C.J.M. Vissenberg, "Charge transport in poly (p -phenylene vinylene) light-emitting diodes", Materials Science and Engineering: R: Reports, ISSN: 0927-796X, 27, 5394(2000).

[23] J. Kalinowski, M. Cocchi, G. Giro, V. Fattori and P.D. Marco, "Injection-controlled electroluminescence in organic light-emitting diodes based on molecularly-doped polymers: II. Double-layer devices", Journal of Physics D Applied Physics, 34, 2274-2281(2001).

[24] Z. Chiguvare, J. Parisi, and V. Dyaknov, "Current limiting mechanisms in indium-tinoxide/poly3-hexylthiophene/aluminum thin film devices", Journal of Applied Physics, 94, 24402448(2003). 
Figures

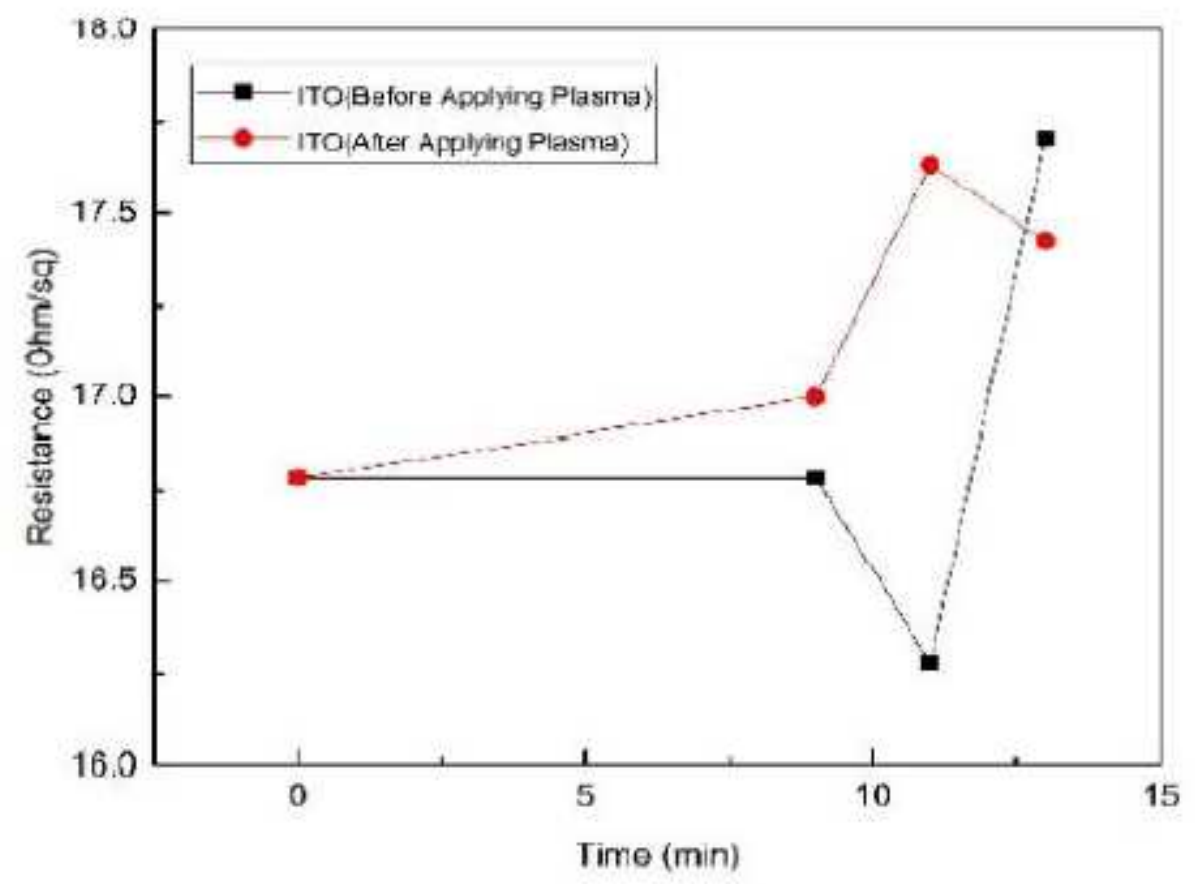

Figure 1

Sheet resistance curve for ITO layers before applying argon atmosphere pressure cold jet plasma, full red circle, and after applying argon atmosphere pressure cold jet plasma, full black circle.

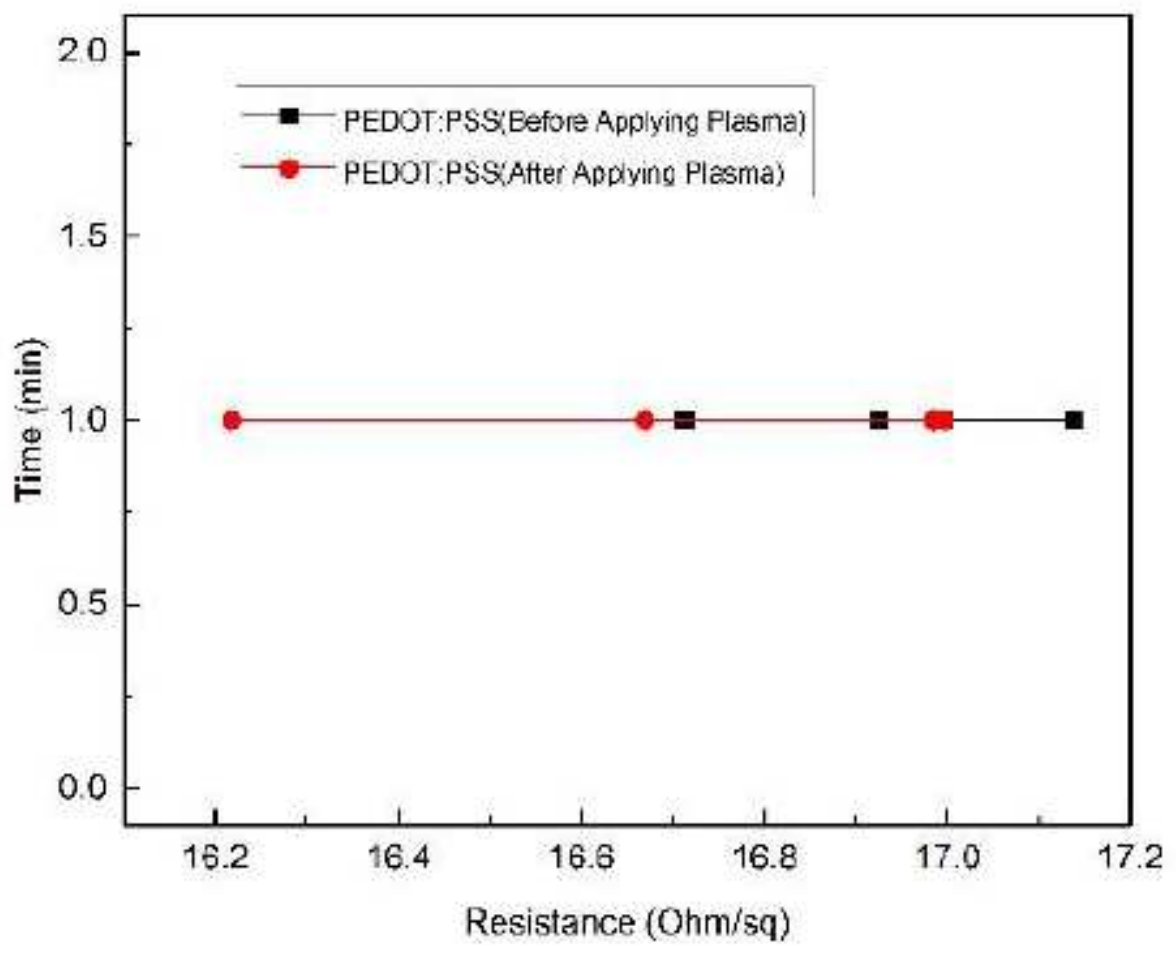


Figure 2

Sheet resistance curve for PEDOT:PSS layers before applying argon atmosphere pressure cold jet plasma, full red circle, and after applying argon atmosphere pressure cold jet plasma, full black circle.

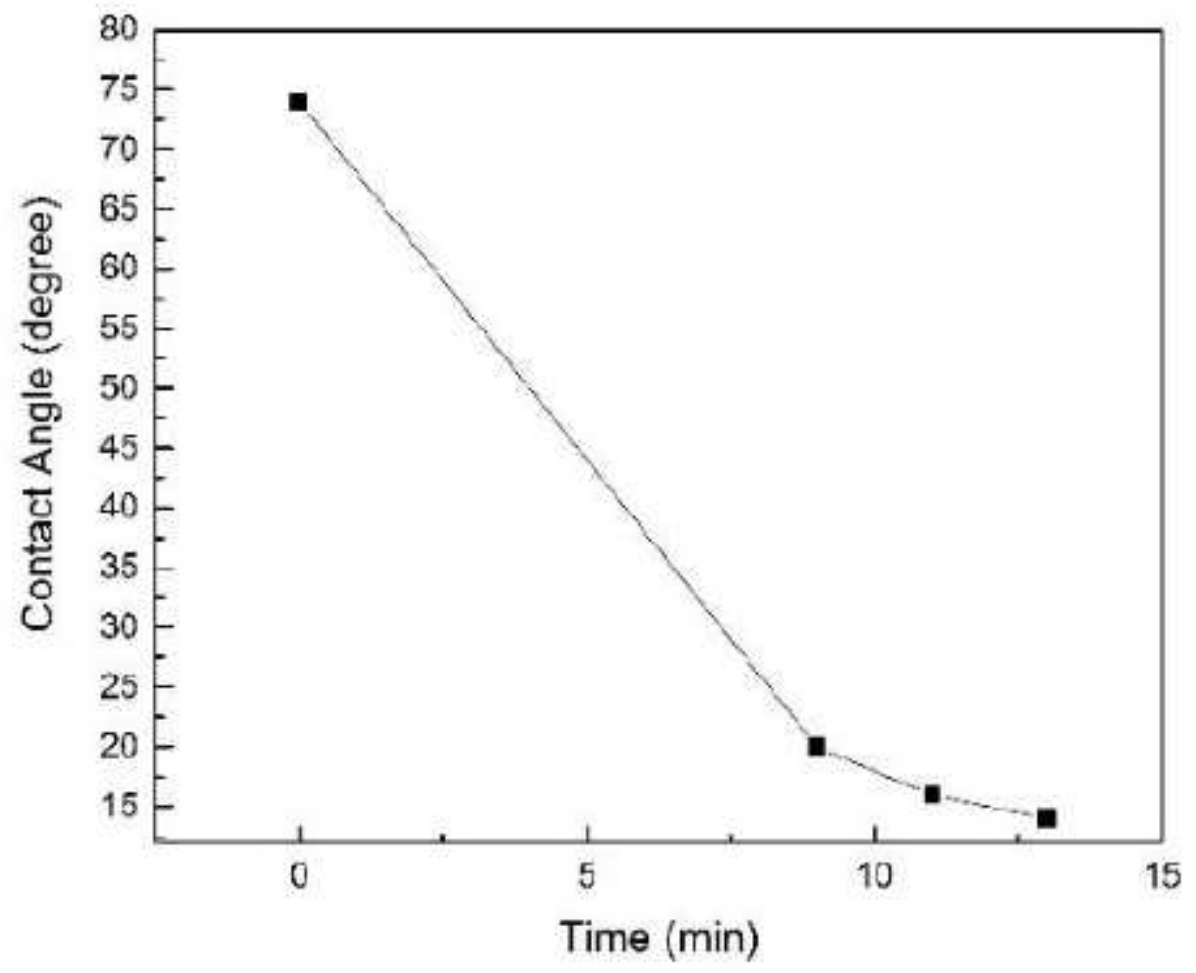

Figure 3

CCD images: Contact angle reducing under applying argon atmosphere pressure cold jet plasma for ITO substrates. 


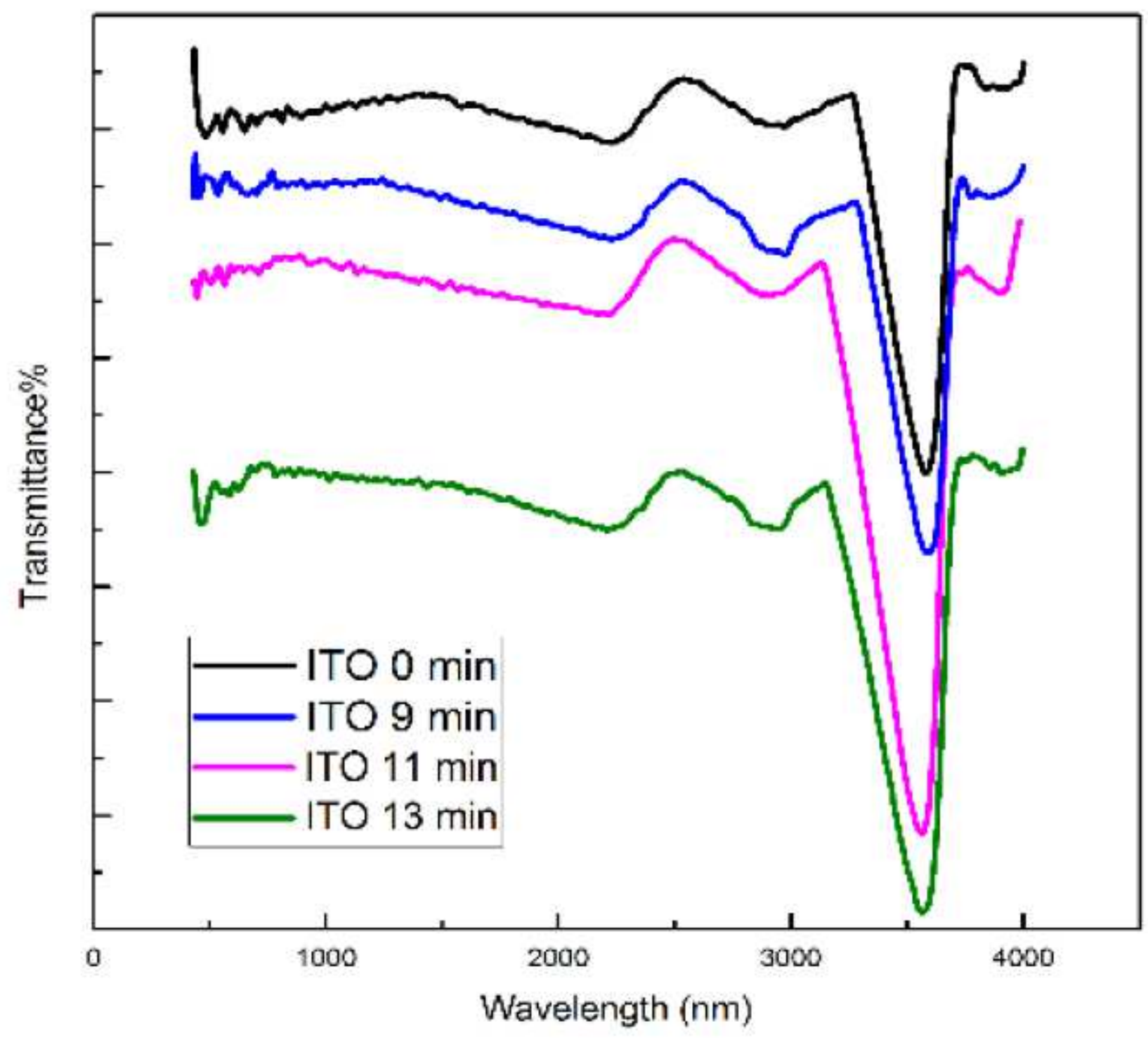

Figure 4

FTIR measurement analyses for ITO layers in various plasma application time. 


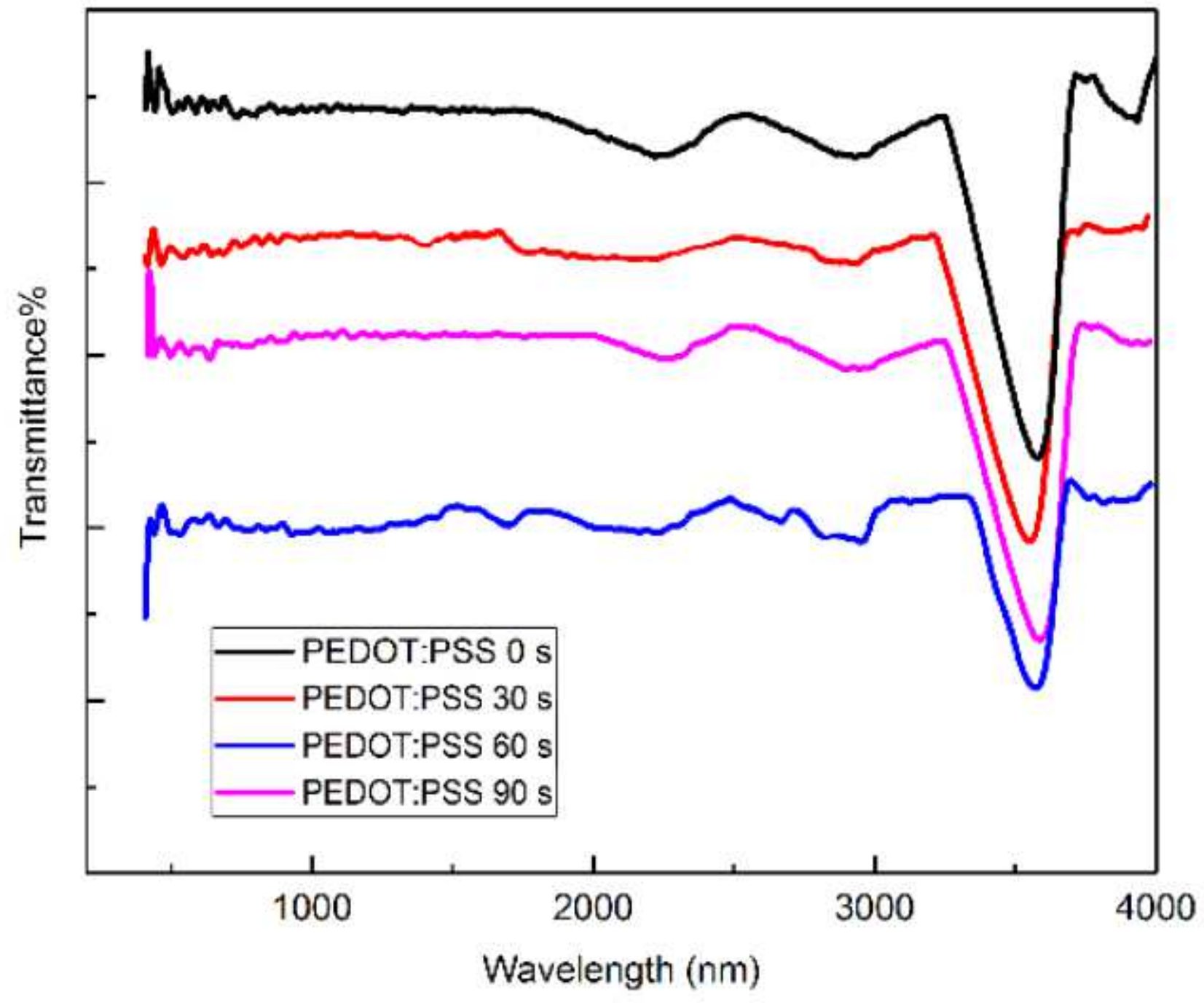

Figure 5

FTIR measurement analyses for PEDOT:PSS layers in various plasma application time. 

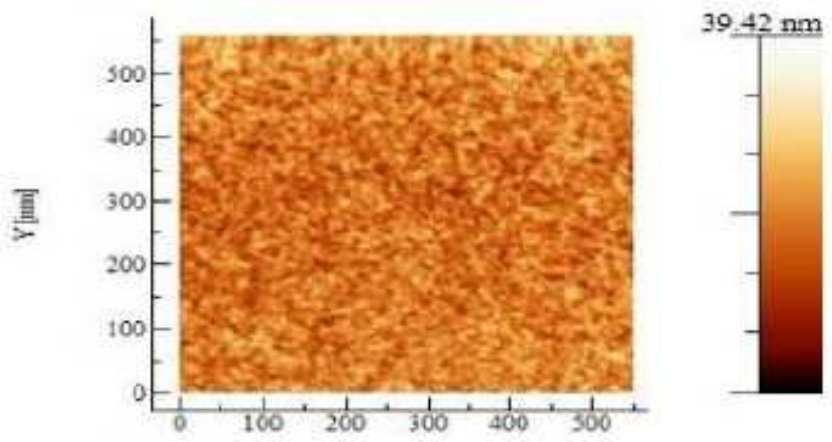

A) ITO(o min plasma)
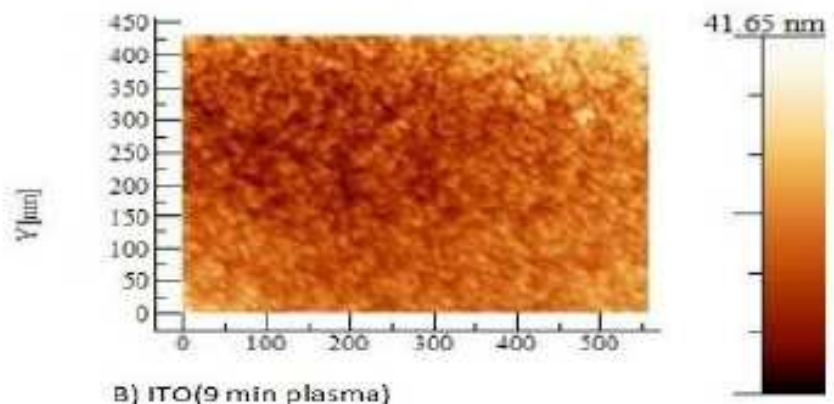

B) 1 TO (9 min plasma)
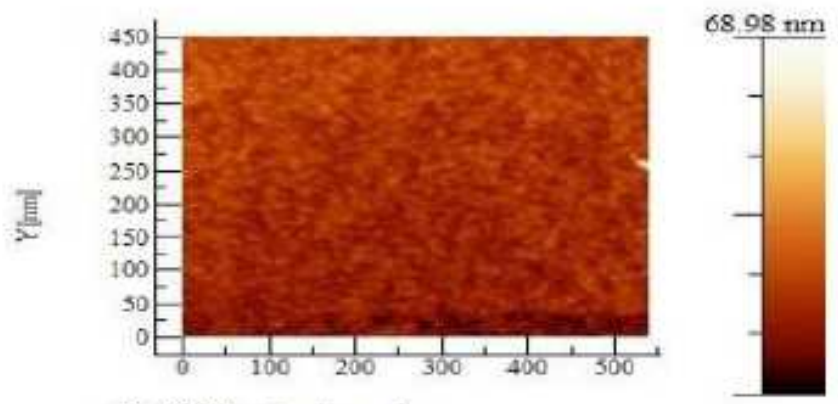

c) $\Pi$ TO(11 min plasma)
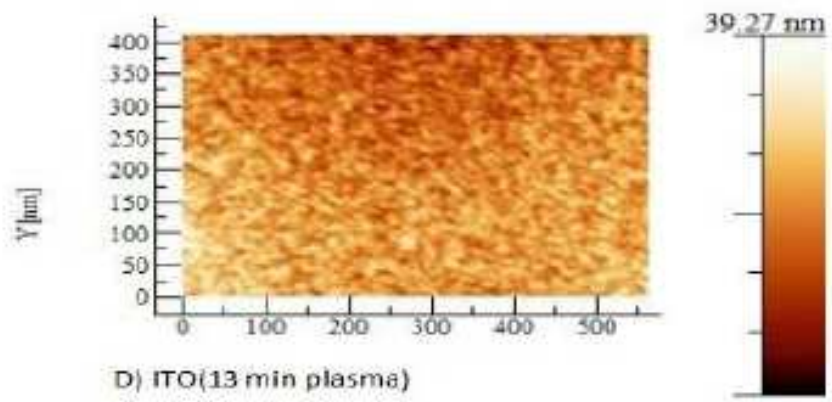

D) ITO(13 min plasma)

$X[\operatorname{tin}]$

\section{Figure 6}

AFM analyses for ITO surface after applying A) 0 minute, B) 9 minute, C) 11 minute, and D) 13 minute argon atmosphere pressure cold jet plasma. 
(A)
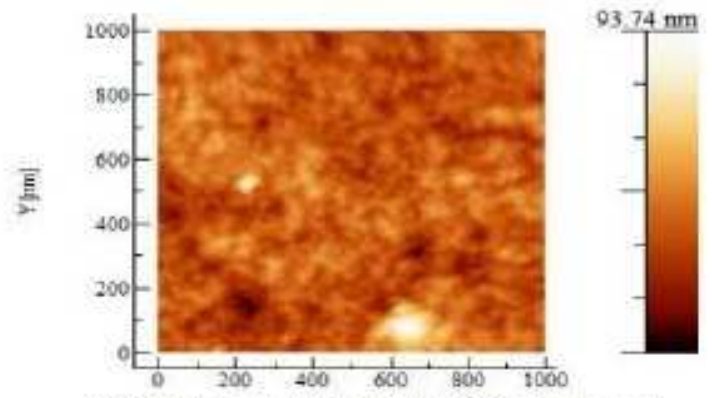

AI) 1 TO(Omin plasma)-PEDOT:PSS(Omin olasma)
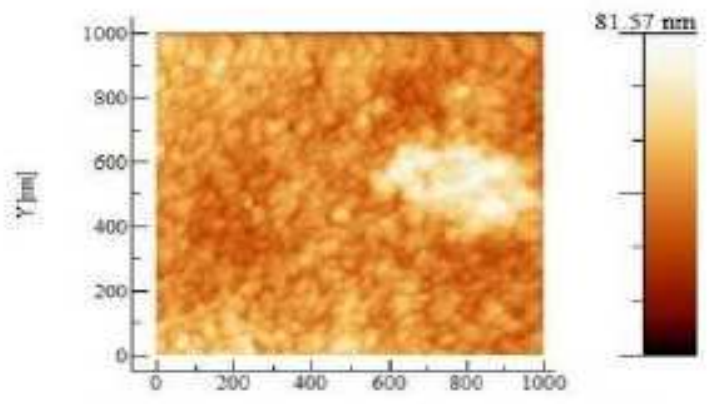

A2) ITO(9min plasma)-PEDOT:PSS(Omin plasma)
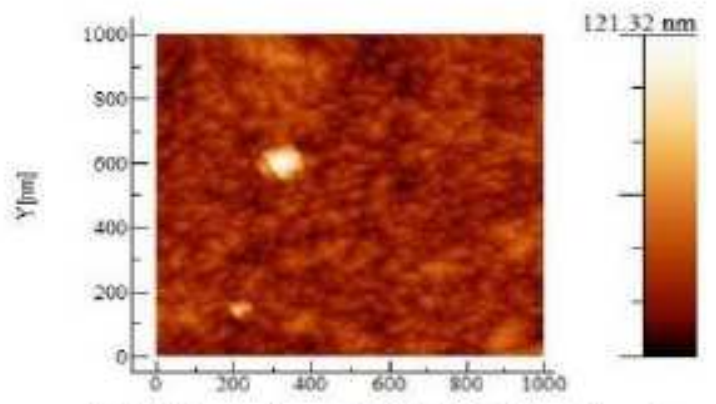

A3) ITO(11min plasma)-PEDOT:PSS\{Omin plasma)
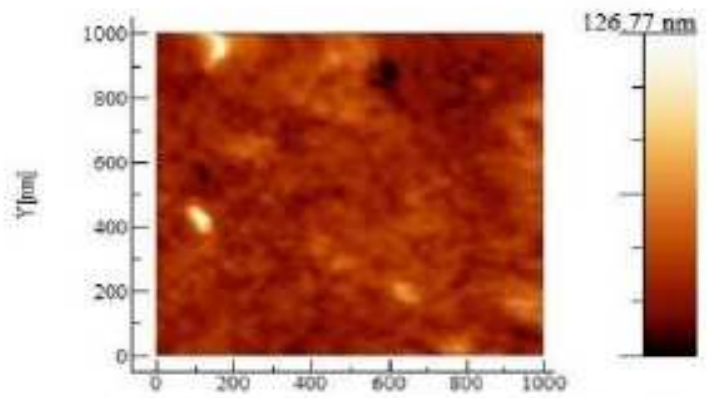

A4) ITO(13min plasma)-PEDOT:PSS(0min plasma)

$\mathrm{X}[\mathrm{n} m]$
(B)

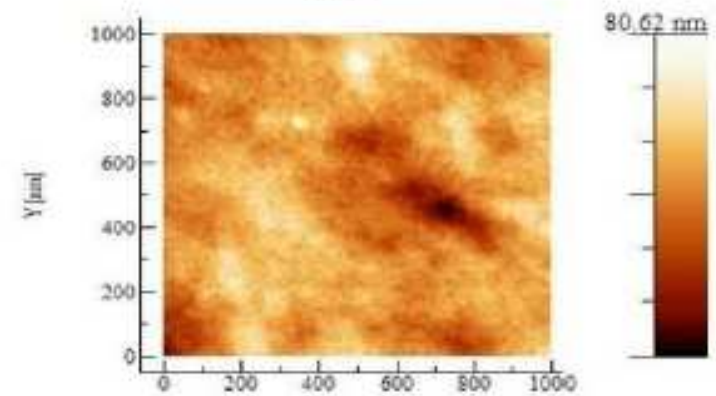

B1) ITO(Omin plasma)-PEDOT:PSS(1min plasma)

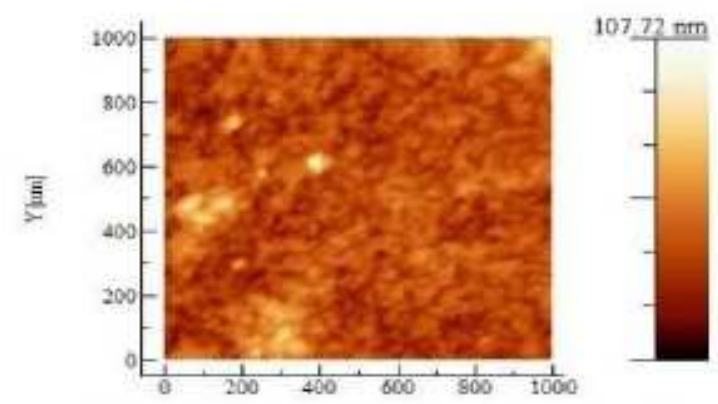

B2) ITO(9min plasma)-PEDOT:PSS(Imin plasma)

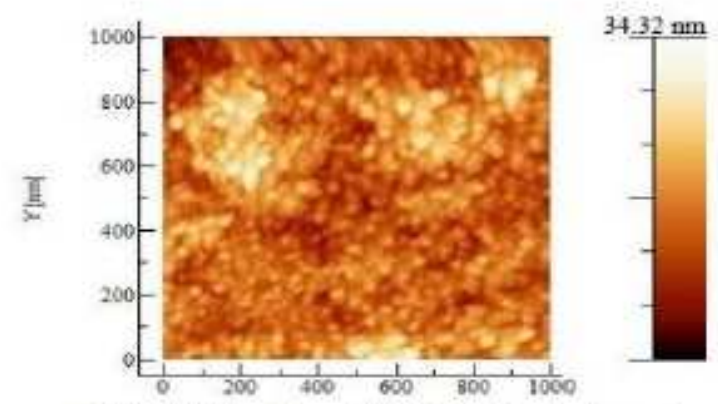

B3) ITO(11 min plasma)-PEDOT:PSS(1min plasma)

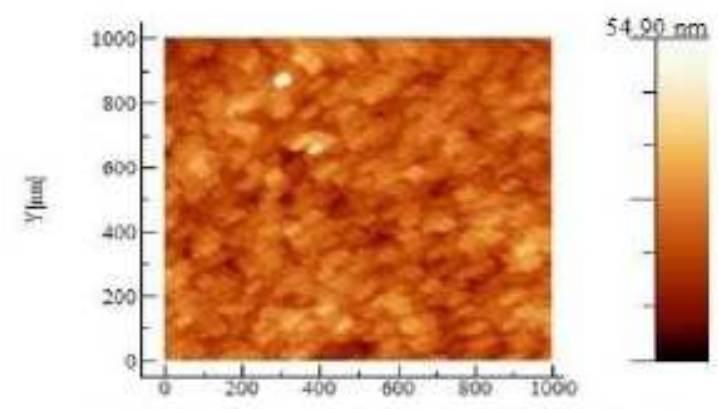

B4) ITO(13min plasma)-PEDOT:PSS[1 min plasma]

Xina]

\section{Figure 7}

AFM analyses for PEDOT:PSS surface before, A1-A4, and after, B1-B4, applying 1 minute argon atmosphere pressure cold jet plasma. 


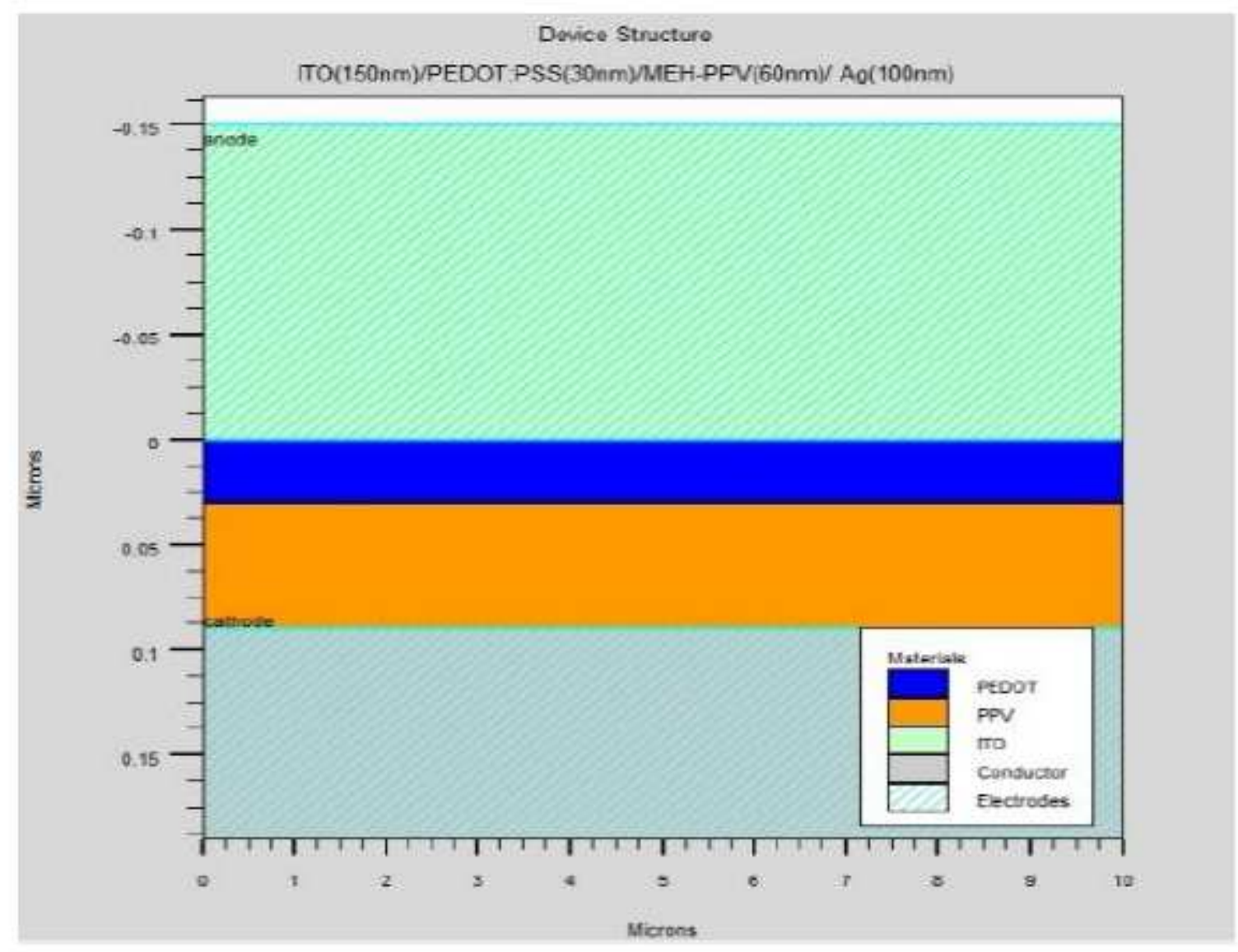

Figure 8

The Device structure consisting of the ITO(150nm)/PEDOT:PSS(30nm)/ MEH-PPV(60nm)/Ag(100nm). 


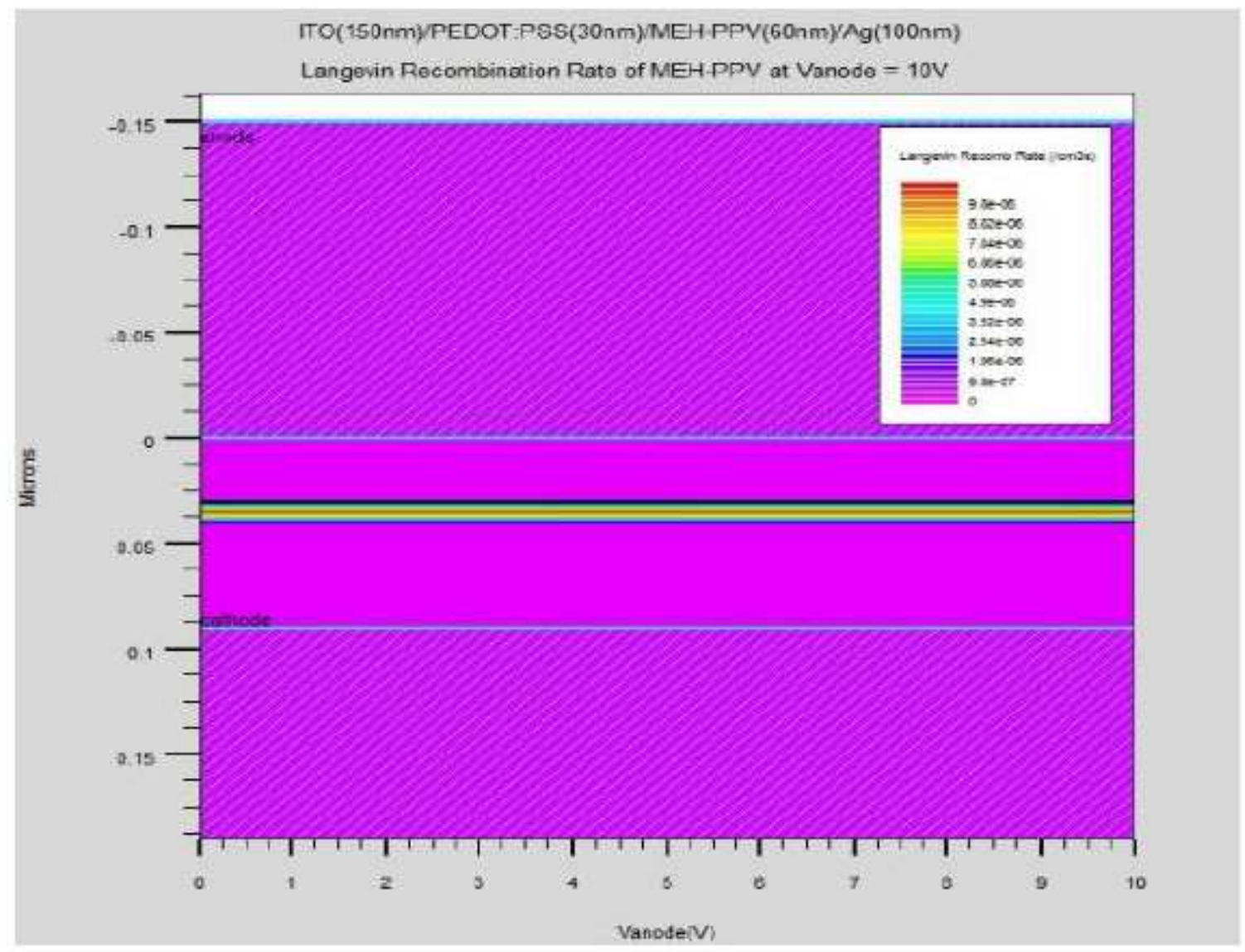

Figure 9

Langvin recombination rate along the various points of the device. 


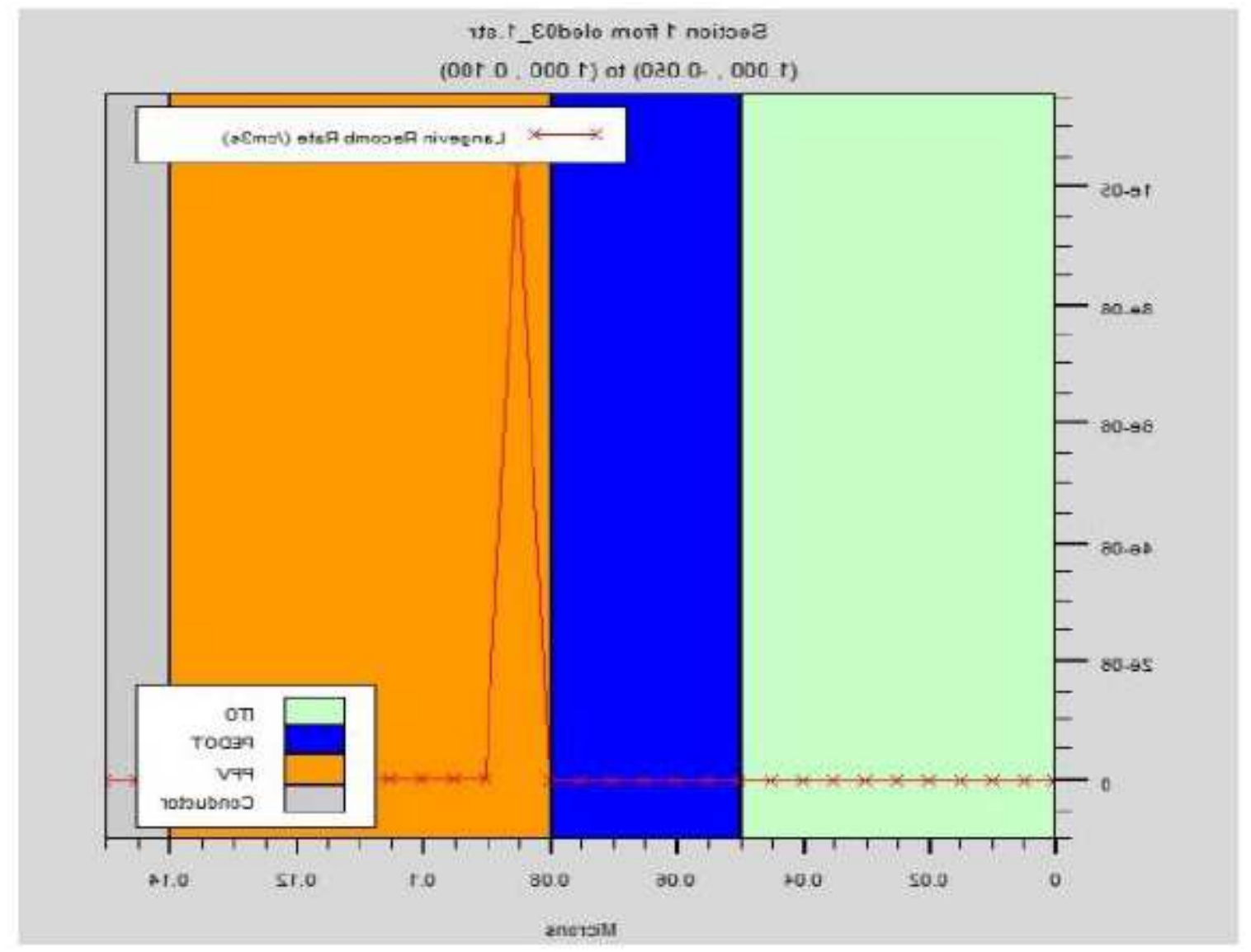

Figure 10

Longvin recombination rate curve in deferent layers of the device.

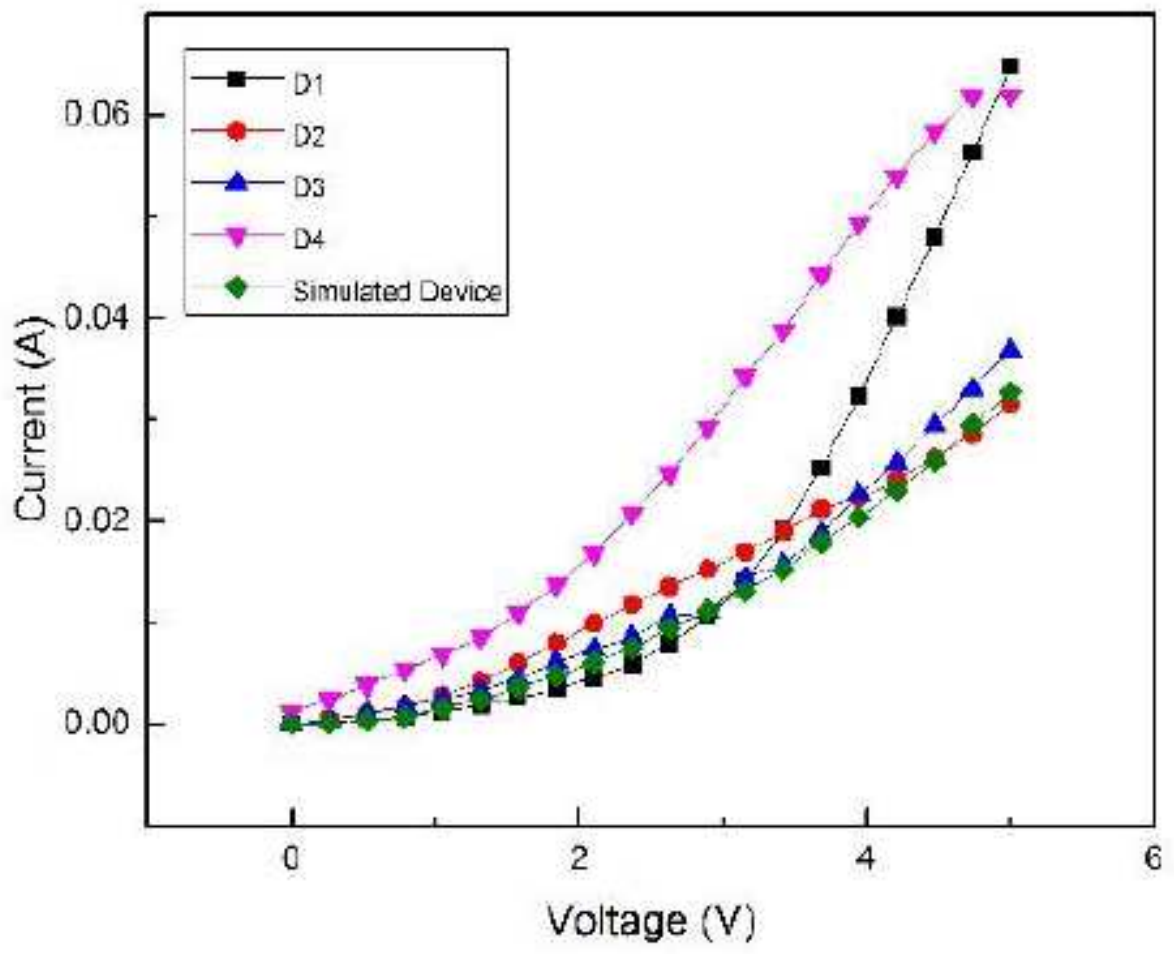




\section{Figure 11}

Current-Voltage characteristics of devices D1, D2, D3, D4, and simulated device with the variation plasma application time.

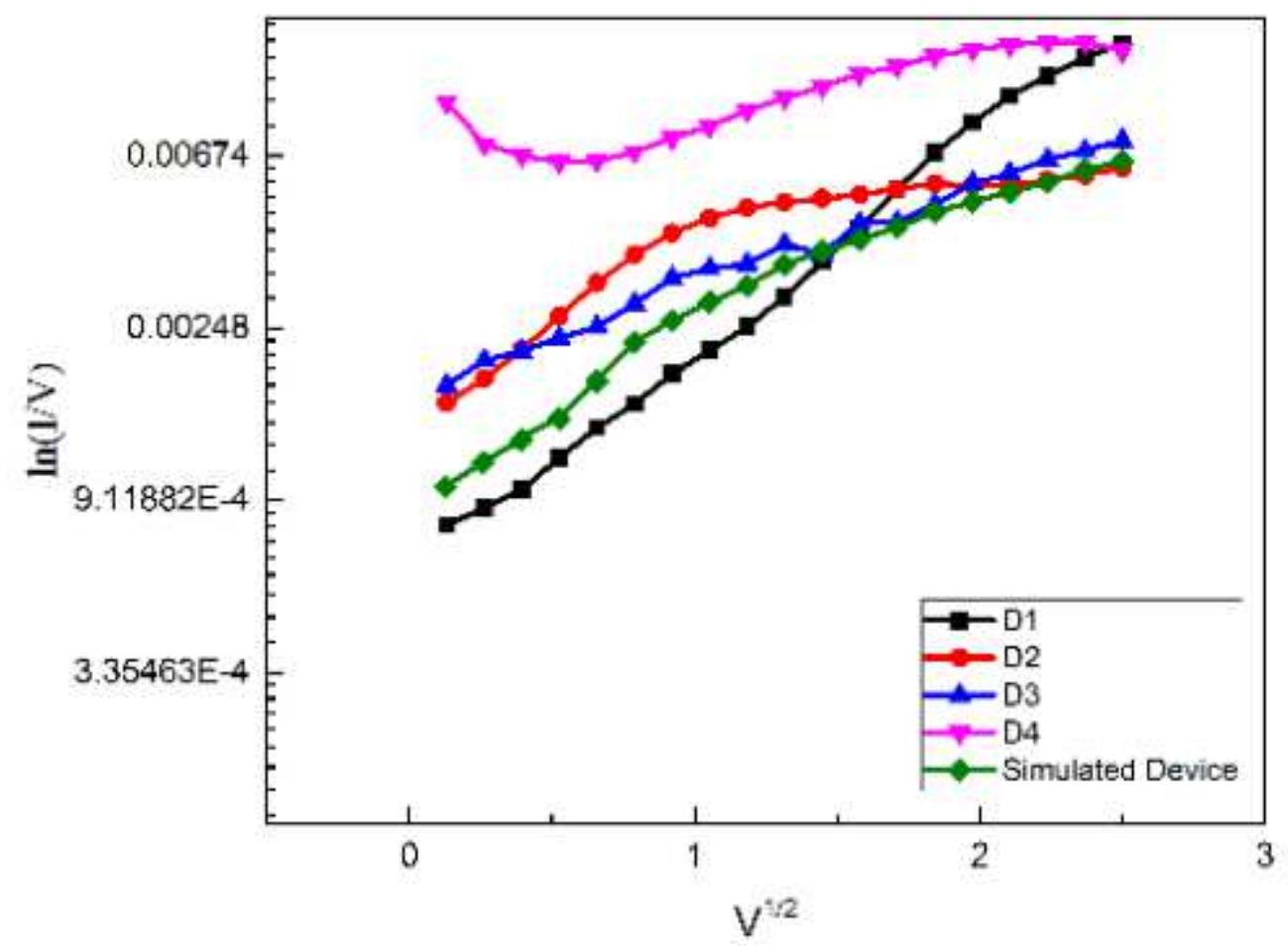

\section{Figure 12}

Dependence of the current density on the applied voltage in D1, D2, D3, D4, and the simulated device, plotted according to the Richardson-Schottky Thermionic Emission model. 


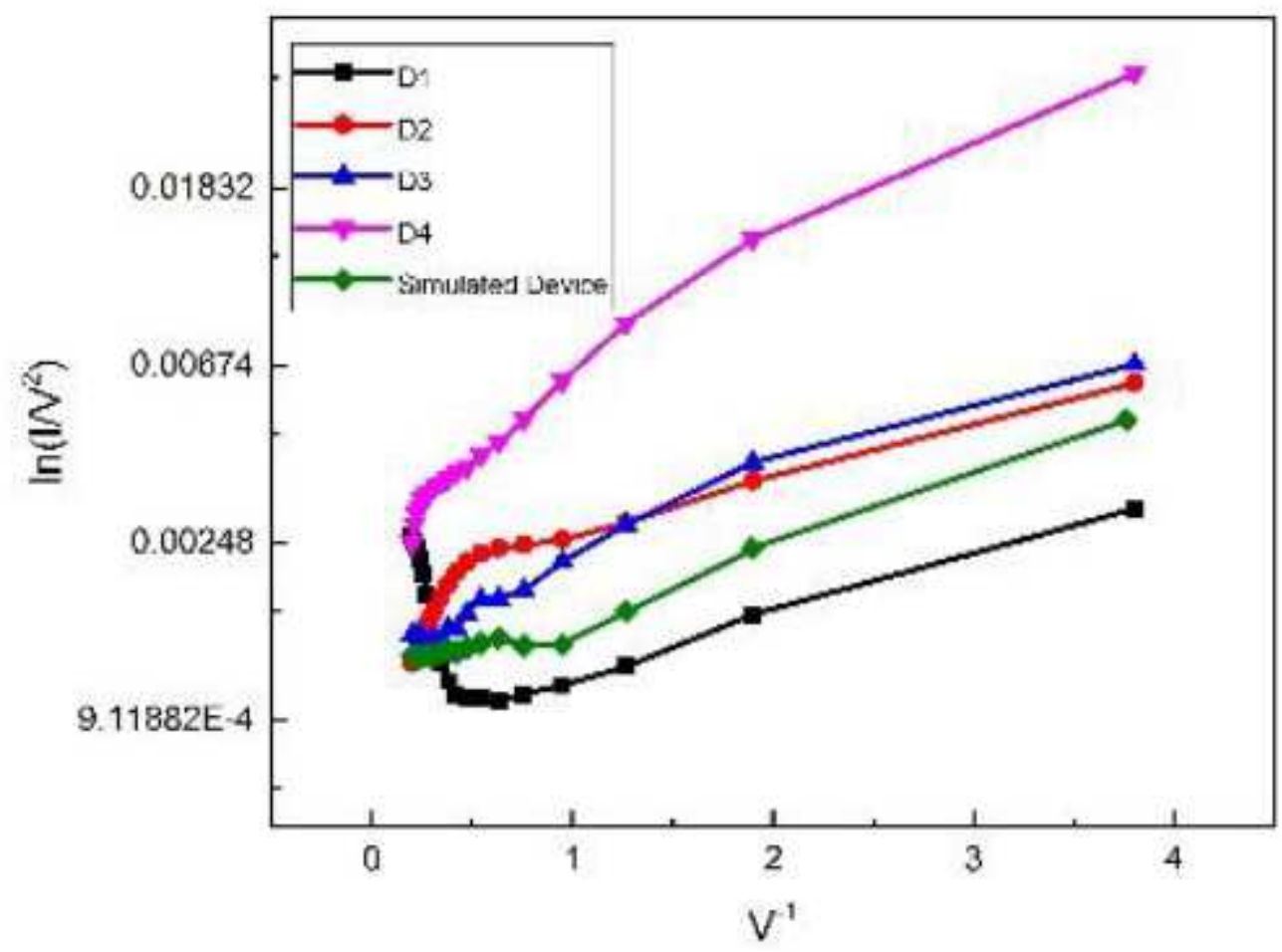

Figure 13

Dependence of the current density on the applied voltage in D1, D2, D3, D4, and simulated device, plotted in the Fowler-Nordheim tunneling emission model. 